\title{
Individual Resource Games and Resource Redistributions
}

\author{
Nicolas Troquard \\ The KRDB Research Centre for Knowledge and Data \\ Faculty of Computer Science \\ Free University of Bozen-Bolzano \\ Piazza Domenicani, 3 \\ I-39100 Bozen-Bolzano BZ, Italy \\ Nicolas. Troquardeunibz.it
}

\begin{abstract}
To introduce agent-based technologies in real-world systems, one needs to acknowledge that the agents often have limited access to resources. They have to seek after resource objectives and compete for those resources.

We introduce a class of resource games where resources and preferences are specified with the language of a resource-sensitive logic. The agents are endowed with a bag of resources and try to achieve a resource objective. For each agent, an action consists in making available a part of their endowed resources. All the resources made available can be used towards the agents' objectives.

We study three decision problems, the first of which is deciding whether an action profile is a Nash equilibrium: when all the agents have chosen an action, it is a Nash Equilibrium if no agent has an incentive to change their action unilaterally.

When dealing with resources, interesting questions arise as to whether some equilibria can be eliminated or constructed by a central authority by redistributing the available resources among the agents. In our economies, division of property in divorce law exemplifies how a central authority can redistribute the resources of individuals, and why they would desire to do so. We thus study two related decision problems:

- rational elimination: given an action profile's outcome, can the endowed resources be redistributed so that it is not the outcome of a Nash equilibrium.

- rational construction: given an action profile's outcome, can the endowed resources be redistributed so that it is the outcome of a Nash equilibrium.

Among other results, we prove that all three problems are PSPACE-complete when the resources are described in the very expressive language of the propositional multiplicative and additive Linear Logic.

We also identify a new modest fragment of Linear Logic that we call MULT, suitable to represent multisets and reason about the inclusion and equality of bags of resources. We show that when the resources are described in MULT, the problem of deciding whether a profile is a Nash equilibrium is in PTIME.
\end{abstract}

\section{Introduction}

Agents, or players, are entities capable of action and trying to reach their goals. In the physical or cyber world, these agents have limited access to resources. They have to seek after resource objectives and compete for those resources. 
This paper makes use of resource-sensitive logics, Linear Logic [16] specifically, to model and solve problems of rational agents interacting in a resource-aware environment. We use Linear Logic to define and reason about a new class of non-cooperative games [30]. Every Linear Logic formula represents a resource. In these games, each agent is endowed with a bag of resources, and has an objective to achieve by transforming the resources made available from the agents' endowed resources. Can we decide whether the resources made available by the agents constitute a Nash equilibrium, that is, whether it is locally optimal under individual strategic considerations? If a local optimal is not desirable, could an arbitrator redistribute the resources in the endowments among the agent so that is not a Nash equilibrium anymore, thus eliminating it? To the contrary, if an outcome is desirable, could an arbitrator redistribute the resources so that is becomes the outcome of a Nash equilibrium, thus constructing it? In this paper we are going to address the computational complexity of the decision problems corresponding to these questions.

As we study the computational complexity of answering these questions about resource-sensitive game theoretical interactions, we will be particularly interested in a few varying parameters:

- What kind of preferences the agents have?

- Do they only care about reaching their resource objectives? (dichotomous)

- Do they also care about how much resource the consume? (parsimonious)

- What is the exact language for talking about resources, and what is the complexity of reasoning about resources in this language?

- Which resource-sensitive logic exactly is used to reason about the resources?

- Can resources be disposed of freely during reasoning? (affine reasoning)

- Must all resources be accounted for during reasoning? (linear reasoning)

This paper is putting together:

1. Linear Logic, which enables the specification of resources and the reasoning about them.

2. Game theory and Nash equilibria, which give us a guideline to characterize normatively good outcome in games whose actions and preferences are defined in terms of the resources expressed in Linear Logic.

3. Computational complexity, which helps us towards an algorithmic treatment of our resource games. It is intimately affected by the precise Linear Logic used to represent the resources.

The models and the algorithms presented here can be used as analytical tools at the disposition of actors and policy makers, for instance in interconnected economies [2, 7]. They can serve at gauging the possible strategic behaviours of the actors and of their competitors, and at identifying possible issues of resource scarcity in a commons.

Our games are reminiscent of notable models existing in the literature. They share the logic-based approach of Boolean games [19, 4]. In Boolean games, each player controls a set of Boolean variables and produces truth values which can be used without restriction towards the Boolean goals. As such, resources proper are absent from Boolean games. Our games also share the resource-sensitiveness of congestion games [39]. In congestion games, the players choose a set of resources (e.g., edges to travel in a graph), and their utility depends on the cost (e.g., delays) of using the shared resources, which depends on the number of players travelling them. Despite some apparent similarities, they are rather superficial. One thing should be obvious: the resources in congestion games are limited to basic resources and lack a rich specification language of resources like the one of resource-sensitive logics.

Using resource-sensitive logic languages to represent goods that are transformed and exchanged between agents owes to previous work, e.g., [17, 33, 34], in multiagent systems and computational social choice [12, 6].

A short version of this paper appeared as [42]. 
Logic: exploiting resource-sensitive logics. In this paper, we study games of resources that are aimed at representing the strategic interactions between rational agents where some combinations of resources replace the abstract notions of action and preferences. In these games, players are endowed with some resources and have preferences upon some resources to be available after the game is played. Players' actions also consist in making available some of the resources they are endowed with.

We propose a class of games of resources that exploits the formalisms and reasoning methods coming from the literature in knowledge representation and computational logics, namely resource-sensitive logics: e.g., Linear Logic, Separation Logic, BI Logic [16, 38, 29]. The languages of these logics allow a fine-grained description of resources, processes, and their harmonious combinations. In computer science, they have been quite successful at modeling systems for multi-party access and modification of shared structures, by allocation and deallocation of resources. The resources used in this paper are not based on a trivial and naïve set theory. Instead, they are based on rich logical languages, supported by elaborate reasoning features.

A resource is represented by one formula of a resource-sensitive logic LOG. More specifically, we assume here that LOG is some propositional variant of Linear Logic. We provide an informal presentation of the resource interpretation of Linear Logic in Section 2 so that the conceptual aspects of the paper can be grasped without a great understanding of Linear Logic.

Game theory: individual resource games. We will consider individual resource games defined formally in Section 3, Each player $i$ of a game will be endowed with a multiset of resources $\epsilon_{i}$. An action for Player $i$ will be to contribute a subset of $\epsilon_{i}$. An (action) profile specifies a contribution for every player. An outcome will be a context consisting of a multiset of resources resulting from a profile. Then, each player $i$ has a goal $\gamma_{i}$, which is a resource, represented by one formula of LOG. An outcome $X$ satisfies the goal of Player $i$ if there is a proof of $X \vdash \gamma_{i}$ in the logic LOG. This will mean that the resources in $X$ can be consumed so as to produce $\gamma_{i} 1$

Intuitively, we can imagine a game taking place around a table. Each player has an objective to create some resource. Each player has also a bag of resources. To play, each player chooses to take some resources (possibly none) from their respective bags and put them on the table in front of them. The outcome is the collection of resources on the table after every player has chosen. A player is satisfied if we can transform the resources on the table so as to produce her goal. It is a Nash equilibrium when no player has an incentive to take back any resources she put on the table, or to add more resources from her bag.

What should be an incentive to take back or to add resources? We will study these games of resources with two kinds of preferences. We will first consider, in Section 4 preferences over outcomes that are dichotomous. We can thus initially say that Player $i$ prefers an outcome $X$ over an outcome $Y$ iff $X \vdash \gamma_{i}$ and $Y \nvdash \gamma_{i}$. Some formal results will lead us to define in Section 5, parsimonious preferences, a finer notion of preference where $i$ may be qualitatively indifferent between $X$ and $Y$, but still prefer $X$ over $Y$ because $i$ 's contribution is strictly less in $X$ than in $Y$.

Algorithms and complexity: solving problems. We will study three decision problems defined also in Section 3, the first of which is deciding whether an action profile is a Nash equilibrium. A Nash equilibrium is, under strategic considerations, a local optimal. A situation in which every agent has picked an action is a Nash equilibrium when no agent has an incentive to change their mind. A variant of this example, with one additional player, will be formalized later in Section 6.3

\footnotetext{
${ }^{1}$ Indeed, $X \vdash \gamma_{i}$ indicates that the resources $X$ are sufficient to produce $\gamma_{i}$, and $X \vdash \gamma_{j}$ indicates that the resources $X$ are sufficient to produce $\gamma_{j}$. It may be however that the resources $X$ are not sufficient to produce $\gamma_{i}$ and $\gamma_{j}$ simultaneously.
} 
Example 1. In a local telecom industry, anti-trust laws forbid a priori cooperation, and regulations oblige the companies to accept traffic from each other. (These telecom companies operate in an interconnected economy [2, 7].) Consider two competing telecommunication companies. Company A manages a $3 G$ network of comprised capacity 3 (bundled as capacities 1 , and 2 ). Company $B$ manages a $4 G$ network of capacity 3 (bundled as capacities 1, and 2). Company A need to offer their customers $3 G$ at capacity 2 and $4 G$ at capacity 1 . Company $B$ need to offer their customers $3 G$ at capacity 2 and $4 G$ at capacity 2.

Activating a network at some capacity has a cost. Companies can privately activate and deactivate networks on the fly. What are the possible equilibria?

There are two Nash equilibria. First, there is the one where Company A provides a bundle of two $3 G$ antennas and Company $B$ provides a bundle of two $4 G$ antennas. Both companies can achieve their goal, and none has an incentive to reduce their contribution as they would not satisfy their goal anymore. Second, there is the one where both Company A and Company B contribute nothing. None of them has an incentive to change their contribution since they would not be able to achieve their goal on their own.

When dealing with resources, interesting questions arise as to whether some equilibria can be eliminated or constructed by a central authority by redistributing the available resources among the players [18]. In the tradition of social mechanism design, redistribution schemes can be used by a central authority to enforce some behavior, either by disincentivizing a behavior or incentivizing a behavior. Formal frameworks dealing with redistribution schemes and economic policies have been studied [11, 25, 28].

Some profiles that are not equilibria can have desirable outcomes. Some equilibria can have outcomes that are undesirable. Desirability must here be understood from the point of view of a system designer. A system designer can redistribute the resources of the players in a game so as to steer the interaction to or away from a particular outcome.

A redistribution consists in reallocating the resources endowed to the players. To every redistribution corresponds a new game where the players maintain their objectives, but their possible actions have changed. If $G^{\epsilon}$ is the original game, and $\epsilon^{\prime}$ is a redistribution of the endowment function $\epsilon$, then $G^{\epsilon^{\prime}}$ is a new game.

We will investigate how resource distribution schemes can contribute to eliminate undesirable game equilibria, and construct desirable game equilibria. They are a form of redistribution of wealth, which consists in wealth being transferred from some individuals to others. In our economies, it exists in the form of social mechanisms such as taxation, public services, and confiscation. Division of property and division of debt in divorce law are good imagery of what a designer can do in the mechanisms we propose in this paper. This example will be formalized later in Section 6.2

Example 2. Ann and Bernard, a couple of bakers, have filed for divorce. Ann is officially the tenant of the business premises of the bakery. Bernard is the owner of the baking equipment. He also owns enough flour to make bread for two years. Ann would like to be able to keep the means of production, and being able to make bread for one year. Bernard wants to keep the shop. In this context, if Ann and Bernard are parsimonious, the outcome is very likely to be the one where Ann does not use the shop and Bernard does not use the breadmaking equipment and the flour. It is the only equilibrium. Neither of them satisfy their objective.

However, an arbitrator can redistribute their endowments. He can give the equipment and half the flour to Ann, and give the shop to Bernard. Doing so, the outcome where Ann and Bernard do not use any of their endowment can be eliminated. Moreover, a new outcome equilibrium can be constructed where both satisfy their objectives.

We will thus look at two decision problems related to Nash equilibria: rational elimination and rational construction of Nash equilibria. 
In a game $G^{\epsilon}$, a profile can be rationally eliminated from a game if there exists a redistribution $\epsilon^{\prime}$ of $\epsilon$ such that there is no profile with the same outcome which is a Nash equilibrium in $G^{\epsilon^{\prime}}$. A profile can be rationally constructed if there exists a redistribution $\epsilon^{\prime}$ such that there is a profile in $G^{\epsilon^{\prime}}$ with the same outcome, which a Nash equilibrium.

Outline. We make a brief presentation of Linear Logic in Section 2 We explain how the language can be used to capture a variety of resources which we will put to use in the remainder of the paper. We present individual resource games formally in Section 3. We also introduce precisely the decision problems NASH EQUILIBRIUM, RATIONAL ELIMINATION, and RATIONAL CONSTRUCTION. We will use and study two kinds of preferences over action profiles. We define dichotomous preferences in Section 4 We study all three decision problems. We propose general algorithms and general complexity results depending on the complexity of sequent provability in LOG, and on whether LOG admits the weakening rule or not (that is, whether LOG is linear or affine). We do the same for parsimonious preferences in Section 5. We also illustrate the decision problems with a few small examples. We present more thorough examples in Section 6 In particular, we formalize Example 2 in Section 6.2 and a variant Example 1 in Section 6.3 and we illustrate the findings of this paper on them. Some concluding remarks are offered in Section 7

We provide a technical appendix. Specifically, Appendix $\mathrm{A}$ presents the sequent rules of the biggest fragment of Linear Logic used in the paper. Appendix B briefly summarizes some elements of computational complexity that can be useful to the reader.

\section{Resources and Linear Logic}

One contribution of this paper is to show that resource-sensitive logics are a useful tool for studying the formal aspects of resources in game theoretical settings. Another contribution is to demonstrate that it is possible to obtain rather general results for a large class of games of resources depending on the formal properties of the logic LOG we start with. This offers the opportunity to tailor a game to the needs of a certain application without changing the framework. We can indeed choose any sensible fragment of a resource-sensitive logic.

We will work with some fragments of Linear Logic [16]. The conceptual aspects of the paper can be grasped without a great understanding of Linear Logic, but the technical results will draw upon the proof theory and its rules presented in the Appendix A A basic understanding of logic is thus necessary to follow the proofs in general, and some intuitions about the resource interpretation of Linear Logic can hopefully contribute to make reading through the remainder of this paper less dull.

\subsection{Formulas and sequents}

A good introduction to Linear Logic and its variants is [41]. We will use logics defined on the language of propositional Linear Logic. The classical tautology splits into the additive $\top$ and the multiplicative 1 . The classical falsum splits into the additive $\mathbf{0}$ and the multiplicative $\perp$. The additive conjunction and disjunction are respectively $\&$ and $\oplus$. The multiplicative conjunction and disjunction are respectively $\not 8$ and $\otimes$. The linear implication is $A \multimap B$ and combines with the multiplicative conjunction such that $(A \otimes(A \multimap B)) \multimap B$ is a valid principle. The linear negation is $\sim A$.

MLL is the multiplicative fragment, whose language is formalized by the grammar $A::=\mathbf{1}|\perp| p|\sim A|$ $A$ \& $A|A \otimes A| A \multimap A$, where $p$ is an atomic formula. It only contains the multiplicative connectives. MALL is the fragment with both additive and multiplicative operators $A::=\top|\mathbf{0}| \mathbf{1}|\perp| p|\sim A| A$ ช $A \mid A \otimes$ $A|A \multimap A| A \& A \mid A \oplus A$. 


\begin{tabular}{|lll|}
\hline$\sim \sim A$ & $\dashv$ & $A$ \\
$\sim(A \& B)$ & $\dashv$ & $(\sim A) \oplus(\sim B)$ \\
$A \ngtr B$ & $\dashv$ & $(\sim A) \multimap B$ \\
$\sim(A \otimes B)$ & $\dashv$ & $(\sim A) \ngtr(\sim B)$ \\
$A \ngtr \perp$ & $\dashv$ & $A$ \\
$A \otimes \mathbf{1}$ & $\dashv$ & $A$ \\
$A \& \top$ & $\dashv$ & $A$ \\
$A \oplus \mathbf{0}$ & $\dashv$ & $A$ \\
$\mathbf{0}$ & $\dashv$ & $\sim \top$ \\
$\perp$ & $\dashv$ & $\sim \mathbf{1}$ \\
\hline
\end{tabular}

Table 1: Remarkable relationship between the Linear Logic connectives. The symbol $\dashv$ indicates provability in both directions.

We now introduce some terminology and notations. A sequent is a statement $\Gamma \vdash \Delta$ where $\Gamma$ and $\Delta$ are finite multisets of occurrences of formulas of LOG. Often, we can conveniently write a multiset $\left\{A_{1}, \ldots, A_{n}\right\}$ as the list of formulas $A_{1}, \ldots, A_{n}$. Also, we use the notation $\Gamma^{*}=\bigotimes_{A \in \Gamma} A$ and $\emptyset^{*}=1$. An intuitionistic sequent is a sequent $\Gamma \vdash A$ with only one formula to the right. Sequent provability will play an important part in the technical work of the paper. A sequent $\Gamma \vdash \Delta$ is provable in LOG if there exists a linear proof using the rules of the logic LOG. Intuitively, $\Gamma \vdash \Delta$ being provable means that the resources in $\Gamma$ can be transformed into either of the resources in $\Delta$. If a sequent $\Gamma \vdash \Delta$ is not provable, we can write $\Gamma \nvdash \Delta$, although we will also often simply write "not $\Gamma \vdash \Delta$ ". Section 2.4 summarizes the computational complexity characterizations of a few fragments of Linear Logic in terms sequent provability.

In the individual resource games introduced in this paper, the action of a player $i$ consists in making available a multiset $C_{i}$ of formulas/resources. The outcome of an action is the multiset union of all the individual actions: $\Gamma=\biguplus_{i} C_{i}$ 2 The goal of a player is a formula/resource $\gamma$. To decide whether the profile with outcome $\Gamma$ satisfies the goal $\gamma$ of a player, we will evaluate the provability of the (intuitionistic) sequent $\Gamma \vdash \gamma$.

The logic captured by all the rules in the Appendix $\mathrm{A}$ is Affine MALL.

A rule that is not part of the calculus is the structural rule of contraction. One rule of contraction (left contraction) says that if something can be proved with two occurrences of $A$, then it can be proved with only one occurrence. Symbolically,

$$
\frac{\Gamma, A, A \vdash \Delta}{\Gamma, A \vdash \Delta} .
$$

This is prohibited in every resource-sensitive logic. Integrating it into Linear Logic, one consequence would be that $A \vdash A \otimes A$. If we interpret formulas as resources-as we do-contraction would be a license to duplicate resources at will. (See [37] for a detailed account of logics without contraction.)

We must concede that some of the connectives of MLL and MALL do not have an intuitive interpretation in terms of resources, in and of themselves. This is the case of the multiplicative and the additive falsums $(\perp, \mathbf{0})$, and of the somehow infamous multiplicative disjunction $\ngtr$. Fortunately, we do not need them to enjoy the full expressivity of Linear Logic. To see that, Table 1 shows how the connectives interact. From it, it is clear that we can as well make without some language redundancy. The resource-

\footnotetext{
${ }^{2}$ We use $\biguplus$ for the multiset union, and $\bigcup$ for the set union.
} 
interpretable language of MLL is

$$
A::=\mathbf{1}|p| \sim A|A \otimes A| A \multimap A,
$$

and the resource-interpretable language of MALL is

$$
A::=\top|\mathbf{1}| p|\sim A| A \otimes A|A \multimap A| A \& A \mid A \oplus A .
$$

It suffices to see the other connectives as definitions, following the equivalences of Table 1 . We define $\perp=\sim \mathbf{1}, \mathbf{0}=\sim \top$, and $A$ ช $B=(\sim A) \multimap B$.

\subsection{Resources as propositions}

A resource captured by a proposition of Linear Logic, can be atomic like one mole of hydrogen $\mathrm{H}$ or one mole of oxygen $\mathrm{O}$. It can be a simultaneous combination of resources, e.g., $\mathrm{O} \otimes \mathrm{O}$ being two moles of oxygen. A resource can be a process transforming resources, e.g., $\mathrm{H}_{2} \mathrm{O} \otimes \mathrm{H}_{2} \mathrm{O} \multimap \mathrm{H}_{2} \otimes \mathrm{H}_{2} \otimes \mathrm{O}_{2}$ would be the well known chemical reaction of electrolysis. It consumes two moles of water to produce two moles of dihydrogen and one mole of dioxygen. Working harmoniously with resources and resource transformation processes with this meticulous control over their combination is made possible using resource-sensitive logics. In a game where a player is endowed with $2 q$ moles of water and a player is endowed with $q$ processes of electrolysis, it is possible to consume these resources and produce $2 q$ moles of hydrogen gas and $q$ of oxygen gas. But not more!

In Section 6.1, we will illustrate our games with an example using chemical reactions. But for the time being, we explain in more details how the refined operators of Linear Logic can be used to formalize and grasp a variety of resources. Table 2 reports possible readings of the connectives.

\begin{tabular}{|ll|}
\hline$A \otimes B$ & $A$ and $B$ simultaneously \\
$A \& B$ & a deterministic choice between $A$ and $B$; not both \\
$A \oplus B$ & $A$ or $B$ non-deterministically; not both \\
$A \multimap B$ & $A$ is sufficient to produce $B$ (losing $A$ in the process) \\
$\mathbf{1}$ & vacuous resource \\
$\top$ & some resource \\
\hline
\end{tabular}

Table 2: Possible resource interpretations of formulas.

Now, whether the occurrence of a resource indicates a consumption or a production of the resources depends on where a formula appears in the sequent. The sequent of Linear Logic

$$
A \vdash B
$$

can be read as

$$
\text { “if you give } A \text { you can receive } B \text { " }
$$

Hence, as it should be expected, we give the resources at the left of the sequent, and receive the resources at the right of the sequent. Table 3 reports possible readings of the sequents. The linear negation allows one to switch the give/receive mode. The sequent $A \vdash \sim B$ represents "give $A$ and $B$, and receive nothing". The sequent $A, \sim B \vdash \perp$ represents "give $A$ and receive $B$ ".

Example 3. A few items can be obtained from vending machine in exchange of money. For instance, giving $\$ 1$ you can choose to receive a chocolate bar or a soft-drink. This is captured by

$$
\$ 1 \vdash \text { chocobar \& drink. }
$$




\begin{tabular}{|ll|}
\hline$\Gamma \vdash A \otimes B$ & receive $A$ and $B$ simultaneously \\
$\Gamma \vdash A \& B$ & choose whether to receive $A$ or $B$; you can't receive both \\
$\Gamma \vdash A \oplus B$ & receive $A$ or $B$; you don't choose; you won't receive both \\
$\Gamma \vdash A \multimap B$ & $\begin{array}{l}\text { receive a resource that can be used in such a way that, if you give } A \text {, you } \\
\text { receive } B \text { (losing } A \text { in the process) }\end{array}$ \\
$A \otimes B \vdash \Delta$ & give $A$ and $B$ simultaneously \\
$A \& B \vdash \Delta$ & choose whether to give $A$ or $B$; you don't give both \\
$A \oplus B \vdash \Delta$ & give $A$ or $B$; you don't choose; you don't give both \\
$A \multimap B \vdash \Delta$ & $\begin{array}{l}\text { give a resource that can be used in such a way that, if you give } A \text {, you } \\
\text { receive } B \text { (losing } A \text { in the process) }\end{array}$ \\
\hline
\end{tabular}

Table 3: Possible resource interpretations of sequents.

Also, giving $\$ 0.8$ you can receive 2 packs of gum. This is captured by:

$$
\$ 0.8 \vdash \text { gum } \otimes \text { gum . }
$$

In the previous example, the formula chocobar\&drink denotes a deliberative choice between chocobar and drink. One and the other can be obtained from $\$ 1$, but not both. This is significantly different from $\$ 1 \vdash$ chocobar $\oplus$ drink which denotes something more akin to the classical disjunction: chocobar or drink can be obtained from $\$ 1$. But for all we know, it might be impossible to actually get one or to get the other, and we don't get to decide.

Example 4. We can represent a simple act of gambling. The sequent

$$
\$ 1 \vdash(\$ 1 \otimes \$ 1) \oplus 1
$$

captures the fact that you can give $\$ 1$ to receive $\$ 2$ or nothing (the vacuous resource); but you don't choose what you get.

The next example uses most of the resource-interpretable connectives.

Example 5. We can capture the fact that $\$ 17$ get you a menu:

$$
\$ 17 \vdash \text { menu . }
$$

The menu consists of a main dish, a side dish, and a dessert:

$$
\text { menu } \vdash \text { dish } \otimes \text { side } \otimes \text { dessert . }
$$

As main dish, you can choose between fish and meat:

$$
\text { dish } \vdash \text { fish \& meat . }
$$

The side dish depends on the season; you don't choose; it is either aubergine, or parsnip with leek, or asparagus:

$$
\text { side } \vdash \text { aubergine } \oplus \text { (parsnip } \otimes \text { leek }) \oplus \text { asparagus . }
$$

Finally, as dessert, you choose between the strudel and the chocolate tart. Moreover, you choose whether to have ice cream for $\$ 1$ extra, or to have no extra (the vacuous resource).

$$
\text { dessert } \vdash(\text { strudel \& chocotart }) \otimes((\$ 1 \multimap \text { icecream }) \& \mathbf{1}) .
$$


We have not illustrated the additive unit $\top$ yet. The next example hints at the upcoming formalization of Example2 in Section 6.2

Example 6. We can formalize the function of the whole baking equipment (mixer, oven, etc) as the resource transformation process flour $\multimap$ bread. That is, the equipment transforms flour into bread. (Arguably ignoring that we would also need water and electricity. For simplicity, water and electricity could here be considered resources that are provably equivalent to the vacuous resource 1.) The sequent

$$
\text { flour, flour, flour } \multimap \text { bread } \vdash \text { bread } \otimes \top
$$

indicates that with two 'tokens' of flour and the breakmaking equipment, one can make bread, and some resources will remain in excess, viz., flour.

The additive unit $\top$ has some connection with the relationship between linear and affine reasoning that we now discuss briefly.

\subsection{Linear vs. affine reasoning and preferences}

Weakening (rules $(W)$ in the Appendix $\mathrm{A}$ ) in the logic LOG can play a crucial role in the satisfaction of the goals of the players. It will also have striking consequences for the algorithmic solutions of the decision problems that we study in this paper.

In the context of resource-sensitive logic, one rule of weakening (left weakening) says that if something can be obtained from a set of resources then it can also be obtained from more resources. Symbolically,

$$
\frac{\Gamma \vdash \Delta}{\Gamma, A \vdash \Delta}
$$

Weakening gives a monotonic flavor to the process of deduction in the logic. Following the terminology in Linear Logic, a logic LOG admitting weakening will be referred to as affine and a logic LOG without weakening will just be referred to as linear.

Despite the fact the Affine Logic admits more inference rules than Linear Logic, the unit $\top$ allows one to simulate the reasoning in Affine Logic with the provability of Linear Logic. Indeed, the sequent $\Gamma \vdash A$ is provable in a logic LOG with the rule of weakening iff the sequent $\Gamma \vdash A \otimes \top$ is provable the logic LOG without using weakening.

In the affine case, $A, B \vdash A$ is a provable sequent. If a player has a goal $\gamma=A$, then she will find her objective satisfied with an outcome $\{A, B\}$. In the linear case, we have in general $A, B \forall A$ (unless $B$ is a vacuous resource equivalent to 1 ). A player with a goal $\gamma=A$ will not be satisfied with an outcome $\{A, B\}$ as she wants $A$ and nothing more. If she is indeed indifferent to leftover resources, her goal can be expressed as $\gamma=A \otimes \top$, when LOG is linear.

Affine logic should be used when extra resources can be disposed of freely. That is, when we can assume that a player satisfied with an outcome would be satisfied with a more sizeable outcome. As we will see in Section 5 this does not prevent players to behave more parsimoniously when they can.

\subsection{Sequent provability and some complexity characterizations}

Given a sequent in a fragment LOG of Linear Logic, the problem of sequent provability (or provability for short) asks whether the sequent is provable from the sequent rules for LOG. When convenient, we write "LOG is in C" when the problem of sequent provability in the logic LOG is in the complexity class C.

Before moving to the technical part of this paper, we quickly summarize the complexity of sequent provability in some fragments and variants of Linear Logic that could be used as the LOG parameter in 
our analysis resource games ${ }^{3}$ The results of this paper will be applicable to every fragment mentioned here. MALL is PSPACE-complete; MLL is NP-complete; Affine MLL is NP-complete; Affine MALL is PSPACE-complete; Intuitionistic MALL is PSPACE-complete; Intuitionistic MLL is NP-complete. Remarkably, and unlike classical logic, these fragments of Linear Logic behave well computationally also in the first-order case. First-Order MLL is NP-complete and First-Order MALL is NEXPTIMEcomplete. See [26, 20].

We will also consider the weaker fragment that we call MULT:

$$
A::=\mathbf{1}|p| A \otimes A
$$

Proposition 7. Sequent provability in Intuitionistic Affine and Intuitionistic Linear MULT is in PTIME.

Proof. Linear MULT is captured by the rules (ax), (cut), (E), $(\otimes \mathrm{R}),(\otimes \mathrm{L}),(1 \mathrm{~L})$, and (1R). Affine MULT also requires $(W)$. To check whether the Intuitionistic sequent $\Gamma \vdash A$ is provable, it suffices to check whether $b^{\bullet}(\Gamma) \supseteq b(A)$ in the case of Affine MULT or $b^{\bullet}(\Gamma)=b(A)$ in the case of Linear MULT, where the flattening functions $b$ and $b^{\bullet}$ are defined as follows:

- $b(\mathbf{1})=\emptyset$

- $b(p)=\{p\}$

- $b(A \otimes B)=b(A) \uplus b(B)$

- $b^{\bullet}(\emptyset)=\emptyset$

- $b^{\bullet}(\{A\} \uplus \Delta)=b(A) \uplus b \bullet(\Delta)$

Both multiset inclusion and multiset equality can be performed in linear time in the number of elements in the sets.

\section{Individual resource games and decision problems}

We formally define our models of individual resource games 4

Definition 8. An individual resource game $(I R G)$ is a tuple $G=\left(N, \gamma_{1}, \ldots, \gamma_{n}, \epsilon_{1}, \ldots, \epsilon_{n}\right)$ where:

- $N=\{1, \ldots, n\}$ is a finite set of players;

- $\gamma_{i}$ is a formula of LOG ( $i$ 's goal, or objective);

- $\epsilon_{i}$ is a finite multiset of formulas of LOG (i's endowment).

Let $G=\left(N, \gamma_{1}, \ldots, \gamma_{n}, \epsilon_{1}, \ldots, \epsilon_{n}\right)$, we define: the set of possible actions of $i$ as the set of multisets $\operatorname{ch}_{i}(G)=\left\{C \mid C \subseteq \epsilon_{i}\right\}$, and the set of profiles in $G$ as $\operatorname{ch}(G)=\prod_{i \in N} \operatorname{ch}_{i}(G)$. When $P=\left(C_{1}, \ldots, C_{k}\right) \in \operatorname{ch}(G)$ and $1 \leq i \leq k$, then $P_{-i}=\left(C_{1}, \ldots, C_{i-1}, C_{i+1}, \ldots, C_{k}\right)$. That is, $P_{-i}$ denotes $P$ without player $i$ 's contribution. The outcome of a profile $P=\left(C_{1}, \ldots, C_{n}\right)$ is given by the multiset of resources out $(P)=\biguplus_{1<i<n} C_{i}$.

We will define " $i$ strongly prefers $\bar{P}$ over $P^{\prime \prime}$ " in due time, reflecting dichotomous preferences first (Section 4) and parsimonious preferences second (Section 5).

Definition 9. Let $G=\left(N, \gamma_{1}, \ldots, \gamma_{n}, \epsilon_{1}, \ldots, \epsilon_{n}\right)$. A profile $P \in \operatorname{ch}(G)$ is a Nash equilibrium iff for all $i \in N$ and for all $C_{i} \in \mathrm{ch}_{i}(G)$, we have that $i$ does not strongly prefer $\left(P_{-i}, C_{i}\right)$ over $P$.

Let us note $N E(G)$ the set of Nash equilibria in $\operatorname{ch}(G)$.

A basic decision problem is the one of determining whether a choice profile is a Nash equilibrium.

\footnotetext{
${ }^{3}$ See Appendix $\mathrm{B}$ for some elements of complexity that will be useful in the proofs in this paper.

${ }^{4}$ Individual resource games were called ideal resource games in [42].
} 
NASH EQUILIBRIUM (NE)

(in) An individual resource game $G$ and $P \in \operatorname{ch}(G)$.

(out) $P \in N E(G)$ ?

Some profiles that are not equilibria can have desirable outcomes. Some equilibria can have outcomes that are undesirable. Hence, it is interesting to investigate how resource distribution schemes influence how undesirable game equilibria can be eliminated and how desirable game equilibria can be constructed.

In the tradition of social mechanism design, redistribution schemes can be used by a central authority to enforce some behavior, either by disincentivizing a behavior or incentivizing a behavior.

We will study redistribution schemes in individual resource games. Let $\epsilon$ be an endowment function such that for every player $i$ we have $\epsilon(i)=\epsilon_{i}$, a multiset of formulas of LOG. A redistribution scheme of $\epsilon$ is an endowment function $\epsilon^{\prime}$ such that

$$
\biguplus_{i \in N} \epsilon(i)=\biguplus_{i \in N} \epsilon^{\prime}(i) .
$$

We note redis $(\epsilon)$ the set of redistributions of the endowment function $\epsilon$.

Given the individual resource game $G^{\epsilon}=\left(N, \gamma_{1}, \ldots, \gamma_{n}, \epsilon(1), \ldots, \epsilon(n)\right)$ we can apply a redistribution scheme where we modify the endowment function $\epsilon$ into $\epsilon^{\prime}$. We thus obtain the individual resource game $G^{\epsilon^{\prime}}=\left(N, \gamma_{1}, \ldots, \gamma_{n}, \epsilon^{\prime}(1), \ldots, \epsilon^{\prime}(n)\right)$.

We will investigate two decision problems inspired by [18], which are related to resource redistributions. We will look at whether the outcome of a resource game can be rationally eliminated. That is whether there is a resource redistribution such that no Nash equilibrium of the new resource game yields this outcome.

RATIONAL ELIMINATION (RE)

(in) An individual resource game $G^{\epsilon}$ and $P \in \operatorname{ch}\left(G^{\epsilon}\right)$.

(out) Is there a redistribution $\epsilon^{\prime}$ of $\epsilon$ such that for all $P^{\prime} \in \operatorname{ch}\left(G^{\epsilon^{\prime}}\right)$, if out $\left(P^{\prime}\right)=\operatorname{out}(P)$ then $P^{\prime} \notin$ $N E\left(G^{\epsilon^{\prime}}\right)$ ?

Conversely, we will look at whether the outcome of a resource game can be rationally constructed. That is whether there is a resource redistribution such that the outcome is the outcome of some Nash equilibrium in the new resource game.

RATIONAL CONSTRUCTION (RC)

(in) An individual resource game $G^{\epsilon}$ and $P \in \operatorname{ch}\left(G^{\epsilon}\right)$.

(out) Is there a redistribution $\epsilon^{\prime}$ of $\epsilon$ such that there is $P^{\prime} \in \operatorname{ch}\left(G^{\epsilon^{\prime}}\right)$ where out $\left(P^{\prime}\right)=\operatorname{out}(P)$ and $P^{\prime} \in N E\left(G^{\epsilon^{\prime}}\right)$ ?

Note that being a game equilibrium is without ambiguity a property of profile. However, after a redistribution of resources in an individual resource game, the space of actions and the space of profiles change. Thus, elimination and construction are more about the outcomes of profiles. Section 4.2 and Section 5.1 will illustrate these decision problems in due time. 


\section{Dichotomous preferences}

Let $G=\left(N, \gamma_{1}, \ldots, \gamma_{n}, \epsilon_{1}, \ldots, \epsilon_{n}\right)$ be an individual resource game. Player $i$, whose goal is $\gamma_{i}$, realizes her objectives in a profile $P$ when out $(P) \vdash \gamma_{i}$. That is, the resources in out $(P)$ can be transformed into a shareable resource $\gamma_{i}$. For $P \in \operatorname{ch}(G)$ and $Q \in \operatorname{ch}(G)$, we say that player $i \in N$ (dichotomously) strongly prefers $P$ over $Q$ (noted $Q \prec_{i} P$ ) iff out $(P) \vdash \gamma_{i}$ and not out $(Q) \vdash \gamma_{i}$.

Proposition 10. Let $G=\left(N, \gamma_{1}, \ldots, \gamma_{n}, \epsilon_{1}, \ldots, \epsilon_{n}\right)$ be an individual resource game, two profiles $P \in$ $\operatorname{ch}(G)$ and $Q \in \operatorname{ch}(G)$, and a player $i \in N$. The problem of deciding whether $Q \prec_{i} P$ is: in $\mathrm{PTIME}$ when provability in $\mathrm{LOG}$ is in $\mathrm{PTIME}$. It is $\mathrm{NP} \wedge$ coNP $=\mathrm{BH}_{2}$-complete when provability in $\mathrm{LOG}$ is NP-complete. It is PSPACE-complete when provability in LOG is PSPACE-complete.

Proof. The language corresponding to the problem is $L=\left\{(P, Q) \mid Q \prec_{i} P\right\}=L_{1} \cap L_{2}$ with $L_{1}=$ $\left\{(P, Q) \mid \operatorname{out}(P) \vdash \gamma_{i}\right\}$, and $L_{2}=\left\{(P, Q) \mid\right.$ not out $\left.(Q) \vdash \gamma_{i}\right\}$. In particular, when the problem of provability in LOG is in NP, we clearly have that $L_{1}$ is a NP language and $L_{2}$ is a coNP language.

For hardness, we consider a newly fabricated decision problem that we call PROV-NONPROV. The problem PROV-NONPROV takes in input two sequents of LOG $\Gamma_{1} \vdash \Delta_{1}$ and $\Gamma_{2} \vdash \Delta_{2}$, and outputs true iff $\Gamma_{1} \vdash \Delta_{1}$ is provable and $\Gamma_{2} \vdash \Delta_{2}$ is not provable. It is easy to see that if LOG is NP-complete, then PROV-NONPROV is $\mathrm{BH}_{2}$-complete, and if LOG is PSPACE-complete, then PROV-NONPROV is PSPACE-complete.

We propose a reduction of PROV-NONPROV into the problem of deciding whether in an individual resource game, a profile is strongly preferred to another profile by a player.

Let $\Gamma_{1} \vdash \Delta_{1}$ and $\Gamma_{2} \vdash \Delta_{2}$ be two sequents of LOG. We can prove using $\perp L, \perp R$, (cut) and (E) that $\Gamma \vdash \Delta$ iff $\Gamma \vdash \Delta, \perp$. Thus, we have $\Gamma_{1} \vdash \Delta_{1}$ iff $\Gamma_{1}, \sim \Delta_{1} \vdash \perp$, and we have $\Gamma_{2} \vdash \Delta_{2}$ iff $\Gamma_{2}, \sim \Delta_{2} \vdash \perp$ 自 Now we construct the game $G=\left(\{1\}, \gamma_{1}=\perp, \epsilon_{1}=\Gamma_{1} \uplus \sim \Delta_{1} \uplus \Gamma_{2} \uplus \sim \Delta_{2}\right)$. It is now easy to see that PROV-NONPROV instantiated with $\Gamma_{1} \vdash \Delta_{1}$ and $\Gamma_{2} \vdash \Delta_{2}$ returns true iff Player 1 strongly prefers $\left(\Gamma_{1} \uplus \sim \Delta_{1}\right)$ over $\left(\Gamma_{2} \uplus \sim \Delta_{2}\right)$ in $G$.

\subsection{Finding Nash equilibria}

We study the complexity of the problem NASH EQUILIBRIUM with dichotomous preferences.

\subsubsection{Hardness}

We are about to prove the lower bound of the complexity NE with dichotomous preferences. Before we do so, observe that by applying the rules $L \sim$ and $R \sim$,

$$
A_{1}, \ldots, A_{n} \vdash B_{1}, \ldots, B_{m} \quad \text { iff } A_{1}, \ldots, A_{n}, \sim B_{2}, \ldots, \sim B_{m} \vdash B_{1}
$$

is immediate. Hence, we can, without loss of generality, consider only the intuitionistic sequents of LOG in the many-to-one reductions of this paper.

Proposition 11. NE is as hard as the problem of checking sequent provability in LOG, even when there is only one player.

Proof. We reduce the problem of sequent provability for the logic LOG. W.l.o.g., we only consider intuitionistic sequents. Let $\Gamma \vdash \delta$ be the intuitionistic sequent where $\Gamma$ is an arbitrary multiset of formulas of LOG and $\delta$ is an arbitrary formula.

We can construct the individual resource game $G$ such that $G=(\{1\}, \delta, \Gamma \cup\{\delta\})$. $G$ is thus the one-player individual resource game where Player 1's goal is to achieve $\delta$, and Player 1 is endowed with

\footnotetext{
${ }^{5}$ For $\Gamma=\left\{A_{1}, \ldots, A_{k}\right\}$ we note $\sim \Gamma$ the set $\left\{\sim A_{1}, \ldots, \sim A_{k}\right\}$.
} 
$\Gamma \cup\{\delta\}$ (this is a set union but we could have chosen the endowment $\Gamma \uplus\{\delta\}$ as well). A profile in $G$ is a choice of Player 1, that is, a subset $C_{1}$ of $\Gamma \cup\{\delta\}$. In this case for any profile $P$ in $G$, out $(P)=P$.

We show that $\Gamma \vdash \delta$ iff $\Gamma \in N E(G)$.

From left to right, suppose that $\Gamma \vdash \delta$. We need to show that $\Gamma \in N E(G)$. That is, for all $C_{1} \subseteq$ $\Gamma \cup\{\delta\}$, if $C_{1} \vdash \delta$ then $\Gamma \vdash \delta$. Since we supposed $\Gamma \vdash \delta$, this is trivially true.

From right to left, suppose that $\Gamma \in N E(G)$. This means that for all $C_{1} \subseteq \Gamma \cup\{\delta\}$, if $C_{1} \vdash \delta$ then $\Gamma \vdash \delta$. Let in particular $C_{1}=\{\delta\}$. Indeed, $C_{1} \subseteq \Gamma \cup\{\delta\}$. Moreover, by (ax) we have $\delta \vdash \delta$. Hence, $\Gamma \vdash \delta$ follows.

\subsubsection{Algorithms}

To establish an upper bound on the complexity of NE let us first outline an algorithm for solving its complement. That is, checking whether a profile is not a Nash equilibrium. Let $P \in \operatorname{ch}(G)$ be a profile. To determine whether $P \notin N E(G)$, we can employ a simple non-deterministic algorithm, showed as Algorithm 1

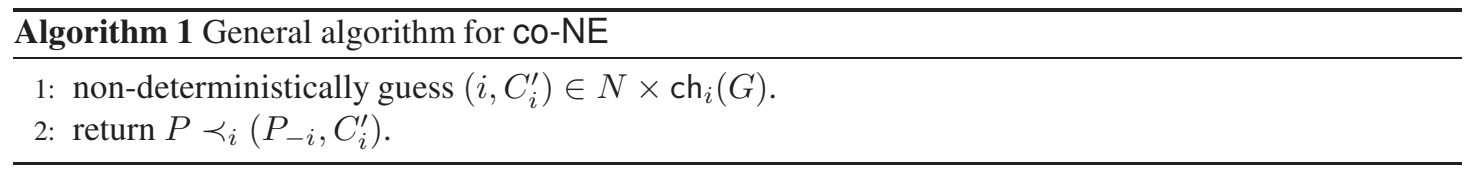

Proposition 12. If the problem of provability in $\mathrm{LOG}$ is in $\mathrm{PTIME}$ then NE is in coNP. If the problem of provability in LOG is in NP then NE is in coNPBH $\mathrm{N}_{2}$ and indeed in $\Pi_{2}^{\mathrm{p}}$. If the problem of provability in LOG is in PSPACE then NE is in PSPACE.

Proof. Consider Algorithm 1 If sequent provability in LOG is in NP, we can check $P \prec_{i}\left(P_{-i}, C_{i}^{\prime}\right)$ in $\mathrm{BH}_{2}$ (Proposition 10). Thus we can check whether $P \notin N E(G)$ in $\mathrm{NP}^{\mathrm{BH}_{2}}$. Finally, we can solve NE in coNP ${ }^{B H_{2}}$. It is the case that $\mathrm{BH}_{2} \subseteq \Delta_{2}^{\mathrm{p}}$, and also that $\mathrm{NP}_{2}^{\mathrm{p}}=\Sigma_{2}^{\mathrm{p}}$ so we can solve NE in $\Pi_{2}^{\mathrm{p}}$. The proofs for the cases of sequent provability in PTIME and PSPACE proceed with similar considerations about Algorithm 1

Affine logic admits the rule of weakening $(W)$, which allows one to discard resources. In this setting, if a player can achieve her goal with the resources $\Gamma$, she can as well achieve her goal with the resources $\Gamma \cup\{A\}$. A consequence is the following lemma, which will have a significant impact on the computational complexity of NE.

Lemma 13. Let $G=\left(N, \gamma_{1}, \ldots, \gamma_{n}, \epsilon_{1}, \ldots, \epsilon_{n}\right)$ be an individual resource game. When LOG is affine, $P \notin N E(G)$ iff $\exists i \in N: P \prec_{i}\left(P_{-i}, \epsilon_{i}\right)$.

Proof. Suppose $P \notin N E(G)$. There is $i \in N$ and $C_{i} \in \operatorname{ch}_{i}(G)$ s.t. $P \prec_{i}\left(P_{-i}, C_{i}\right)$. By definition, out $\left(\left(P_{-i}, C_{i}\right)\right) \vdash \gamma_{i}$ and out $(P) \forall \gamma_{i}$. We have $C_{i} \subseteq \epsilon_{i}$, so by applying weakening $(W)$ with every instance of formulas in $\epsilon_{i} \backslash C_{i}$, we can prove that out $\left(\left(P_{-i}, \epsilon_{i}\right)\right) \vdash \gamma_{i}$. We thus have that there is $i \in N$ s.t. $P \prec_{i}\left(P_{-i}, \epsilon_{i}\right)$. The other way around is immediate from the definition of Nash equilibria.

It means that, in a profile, if no player has an incentive to deviate by making available their whole endowment, then the profile is a Nash equilibrium. The very profile where all the players make available their whole endowment is trivially such a profile. The next proposition follows immediately:

Proposition 14. Let $G=\left(N, \gamma_{1}, \ldots, \gamma_{n}, \epsilon_{1}, \ldots, \epsilon_{n}\right)$ be an individual resource game. When LOG is affine: $N E(G) \neq \emptyset$ and $\left(\epsilon_{1}, \ldots, \epsilon_{n}\right) \in N E(G)$.

Lemma 13 also helps us to establish the following result. 
Proposition 15. When LOG is affine, if the problem of sequent provability in LOG is in PTIME then NE is in PTIME. If the problem of sequent provability in $\mathrm{LOG}$ is in NP then $\mathrm{NE}$ is in $\mathrm{P} \mathrm{NP} \|$. If the problem of sequent provability in LOG is in PSPACE then NE is in PSPACE.

Proof. Let $G=\left(N, \gamma_{1}, \ldots, \gamma_{n}, \epsilon_{1}, \ldots, \epsilon_{n}\right)$ be an individual resource game and let $P \in \operatorname{ch}(G)$ be a profile. One can check whether $P \in N E(G)$ with Algorithm 2

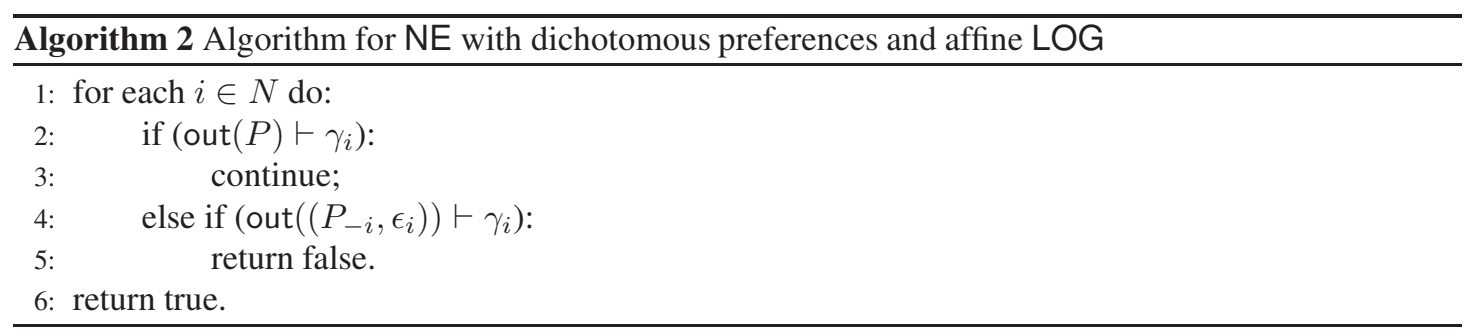

For correctness, note that the instructions of the lines $2-4$ are equivalent to a test of whether out $(P) \forall$ $\gamma_{i}$ and out $\left(\left(P_{-i}, \epsilon_{i}\right)\right) \vdash \gamma_{i}$, that is, $P \prec_{i}\left(P_{-i}, \epsilon_{i}\right)$. Lemma13 ensures that exactly when there is an $i \in N$ such that $P \prec_{i}\left(P_{-i}, \epsilon_{i}\right)$ we can conclude that $P$ is not a Nash equilibrium.

Suppose sequent provability in LOG is in NP. The algorithm can be simulated by a deterministic oracle Turing machine in polynomial time with $2 n$ non-adaptive queries to an NP oracle. Indeed, $P \in$ $N E(G)$ is thus a $\mathrm{P}^{\mathrm{NP} \|[2 n]}$ predicate. The problem is in $\mathrm{P}^{\mathrm{NP} \|}$. When sequent provability in LOG is in PTIME (resp., PSPACE), the algorithm runs in polynomial time (resp., polynomial space).

\subsection{Elimination}

A very simple illustration of RATIONAL ELIMINATION is given by the individual resource game $G^{\epsilon}=$ $\left(\{1,2\}, \gamma_{1}=B, \gamma_{2}=A,\{A\},\{B\}\right)$. There are two players. Player 1 wants $B$ but is endowed with $\{A\}$, while Player 2 wants $A$ but is endowed with $\{B\}$. The game $G^{\epsilon}$ can be represented as on Figure1. (We indicate the realized objectives assuming that LOG is affine.)

\begin{tabular}{|c|c|c|}
\hline $1 \quad 2$ & $\emptyset$ & $\{B\}$ \\
\hline$\emptyset$ & $\emptyset \square$ & $\{B\} \square: \gamma_{1}$ \\
\hline$\{A\}$ & $\{A\} \square: \gamma_{2}$ & $\{A, B\} \square: \gamma_{1}, \gamma_{2}$ \\
\hline
\end{tabular}

Figure 1: The game $G^{\epsilon} \cdot \gamma_{1}$ and $\gamma_{2}$ indicate that Player 1 and Player 2 have their goals satisfied, assuming LOG is affine. The symbol $\square$ denotes a Nash equilibrium.

One can readily check that all profiles are Nash equilibria. However, the profile $(\{A\},\{B\})$ is more 'socially desirable' than the others since it satisfies both players' goal.

A centralized authority could effectively eliminate the others by redistributing the resources present in $G^{\epsilon}$ so as to obtain $G^{\epsilon^{\prime}}=\left(\{1,2\}, \gamma_{1}=B, \gamma_{2}=A,\{B\},\{A\}\right)$. The game $G^{\epsilon^{\prime}}$ can be represented as on Figure 2

The only Nash equilibrium is now the one with outcome $\{B, A\}$.

\subsubsection{Algorithms}

As a consequence of Proposition 14, we already know that: 


\begin{tabular}{|c|c|c|}
\hline $1 \quad 2$ & $\emptyset$ & $\{A\}$ \\
\hline$\emptyset$ & $\emptyset$ & $\{A\}: \gamma_{2}$ \\
\hline$\{B\}$ & $\{B\}: \gamma_{1}$ & $\{A, B\} \square: \gamma_{1}, \gamma_{2}$ \\
\hline
\end{tabular}

Figure 2: The game $G^{\epsilon^{\prime}}$.

Proposition 16. Let $G=\left(N, \gamma_{1}, \ldots, \gamma_{n}, \epsilon_{1}, \ldots, \epsilon_{n}\right)$ be an individual resource game. When LOG is affine, the profile $P$ such that out $(P)=\biguplus_{j} \epsilon_{j}$ is not rationally eliminable.

This is very specific to the affine case (and dichotomous preferences), and even then, it is of course not true of all Nash equilibria. To decide whether some outcome is rationally eliminable, one naïve approach consists in trying all possible redistributions and check whether the outcome is a Nash equilibrium in the resulting individual resource game. Instead, we are going to exploit a pleasant property, analogous to [18, Corollary 4].

Let $G^{\epsilon}=\left(N, \gamma_{1}, \ldots, \gamma_{n}, \epsilon(1), \ldots, \epsilon(n)\right)$ be an individual resource game. For each player $i \in N$, we define $G^{[\epsilon \triangleright i]}$ where $[\epsilon \triangleright i]$ is the redistribution of $\epsilon$ where all resources are assigned to $i$, that is:

$$
[\epsilon \triangleright i](j)= \begin{cases}\biguplus_{k \in N} \epsilon(k) & \text { when } j=i \\ \emptyset & \text { otherwise. }\end{cases}
$$

Because there is only one active player in $G^{[\epsilon \triangleright i]}$, we will sometimes write a profile of $G^{[\epsilon \triangleright i]}$ as $\left(C_{i}\right)$ with $C_{i} \in \operatorname{ch}_{i}\left(G^{[\epsilon \triangleright i]}\right)$ instead of $\left(\emptyset, \ldots, \emptyset, C_{i}, \emptyset, \ldots, \emptyset\right)$, by abuse of notation.

Lemma 17. Let $G^{\epsilon}$ be an individual resource game and $P \in \operatorname{ch}\left(G^{\epsilon}\right)$. $P$ is rationally eliminable iff there is a player $i \in N$ and a profile $Q \in \operatorname{ch}\left(G^{[\epsilon \triangleright i]}\right)$, such that $\operatorname{out}(Q)=\operatorname{out}(P)$ and $Q \notin N E\left(G^{[\epsilon \triangleright i]}\right)$.

Proof. From right to left. Suppose $Q \notin N E\left(G^{[\epsilon \triangleright i]}\right)$ for some $i \in N$. Let also $P \in \operatorname{ch}\left(G^{\epsilon}\right)$ be a profile and assume out $(P)=\operatorname{out}(Q)$. When there is at most one player with a non-empty endowment, as in $[\epsilon \triangleright i]$, there is a one-to-one correspondence between the set of profiles and the set of outcomes. Thus, there is one and only one profile in $G^{[\epsilon \triangleright i]}$ with outcome out $(P)$ and it is $Q$. So there is a redistribution of $\epsilon$, namely $[\epsilon \triangleright i]$, such that for all profiles $Q \in \operatorname{ch}\left(G^{[\epsilon \triangleright i]}\right)$ with outcome out $(P)$, we have $Q \notin$ $N E\left(G^{[\epsilon \triangleright i]}\right)$. So $P$ is rationally eliminable.

From left to right. Suppose that $P$ is rationally eliminable. Thus, there is a redistribution $\epsilon^{\prime}$ of $\epsilon$ such that for all $P^{\prime} \in \operatorname{ch}\left(G^{\epsilon^{\prime}}\right)$, if out $\left(P^{\prime}\right)=\operatorname{out}(P)$ then $P^{\prime} \notin N E\left(G^{\epsilon^{\prime}}\right)$. So let $R \in \operatorname{ch}\left(G^{\epsilon^{\prime}}\right)$ be an arbitrary profile with out $(R)=\operatorname{out}(P)$. By assumption, we have that $R \notin N E\left(G^{\epsilon^{\prime}}\right)$. By definition of Nash equilibria, this means that there is $i \in N$ and $C_{i}^{\prime} \in \operatorname{ch}_{i}\left(G^{\epsilon^{\prime}}\right)$ such that $R \prec_{i}\left(R_{-i}, C_{i}^{\prime}\right)$. Now consider the game $G^{[\epsilon \triangleright i]}$. We have out $(R) \in \operatorname{ch}_{i}\left(G^{[\epsilon \triangleright i]}\right)$ and out $\left(\left(R_{-i}, C_{i}^{\prime}\right)\right) \in \operatorname{ch}_{i}\left(G^{[\epsilon \triangleright i]}\right)$. Let the profile $R^{1} \in \operatorname{ch}\left(G^{[\epsilon \triangleright i]}\right)$ with $R_{i}^{1}=\operatorname{out}(R)$ and $R_{j}^{1}=\emptyset$ when $j \neq i$. Let $R^{2} \in \operatorname{ch}\left(G^{[\odot \triangleright i]}\right)$ be the profile with $R_{i}^{2}=\operatorname{out}\left(\left(R_{-i}, C_{i}^{\prime}\right)\right)$ and $R_{j}^{2}=\emptyset$ when $j \neq i$. Since, $R \prec_{i}\left(R_{-i}, C_{i}^{\prime}\right)$, we also have $R^{1} \prec_{i} R^{2}$. So $R^{1} \notin N E\left(G^{[\epsilon \triangleright i]}\right)$. The profile $R^{1}$ is the only profile of $G^{[\epsilon \triangleright i]}$ with outcome out $(P)$. So we can conclude.

We establish an upper bound on the complexity of RE when LOG does not admit the weakening rule.

Proposition 18. When LOG is linear, RE is in $\mathrm{NP}$ when provability in $\mathrm{LOG}$ is in $\mathrm{PTIME}$, in $\mathrm{NP}^{\mathrm{BH}_{2}}$ and indeed in $\sum_{2}^{\mathrm{p}}$ when LOG is in NP, and in PSPACE when LOG is in PSPACE. 


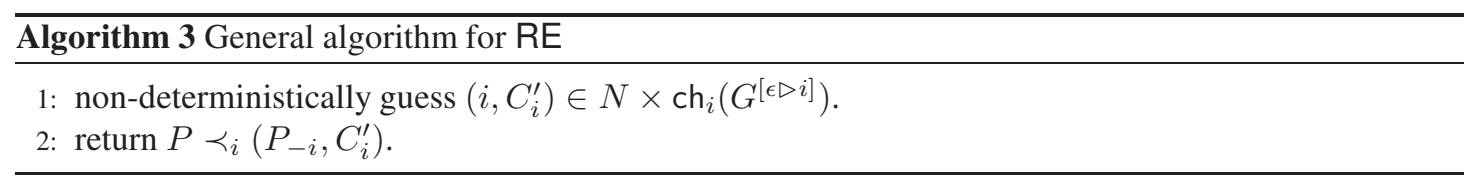

Proof. Let $P \in \operatorname{ch}\left(G^{\epsilon}\right)$ be a profile. To determine whether $P$ is rationally eliminable, we can use Algorithm 3 .

Straightforwardly, it guesses a player $i$ and a deviation in the game $G^{[\epsilon \triangleright i]}$ for Player $i$ from the profile $($ out $(P)) \in \operatorname{ch}\left(G^{[\epsilon \triangleright i]}\right)$, and checks whether Player $i$ has an incentive to do this deviation. By Lemma 17 if such a player and deviation exist and only if they exist, the profile $P$ is rationally eliminable in $G^{\epsilon}$. So the algorithm is correct. It can of course be simulated by a non-deterministic oracle Turing machine with one call to an oracle for $P \prec_{i}\left(P_{-i}, C_{i}^{\prime}\right)$. Proposition 10 informs us of a containing class of this oracle.

When LOG admits the weakening rule, we can propose a surprisingly simple algorithm, which takes advantage of both Lemma 13 and Lemma 17

Proposition 19. When $\mathrm{LOG}$ is affine, RE is in $\mathrm{PTIME}$ when provability in $\mathrm{LOG}$ is in $\mathrm{PTIME}$, in $\mathrm{P}^{\mathrm{NP} \|}$ when LOG is in NP, and in PSPACE when LOG is in PSPACE.

Proof. Let $G=\left(N, \gamma_{1}, \ldots, \gamma_{n}, \epsilon_{1}, \ldots, \epsilon_{n}\right)$ be an individual resource game and let $P \in \operatorname{ch}(G)$ be a profile. Consider Algorithm 4

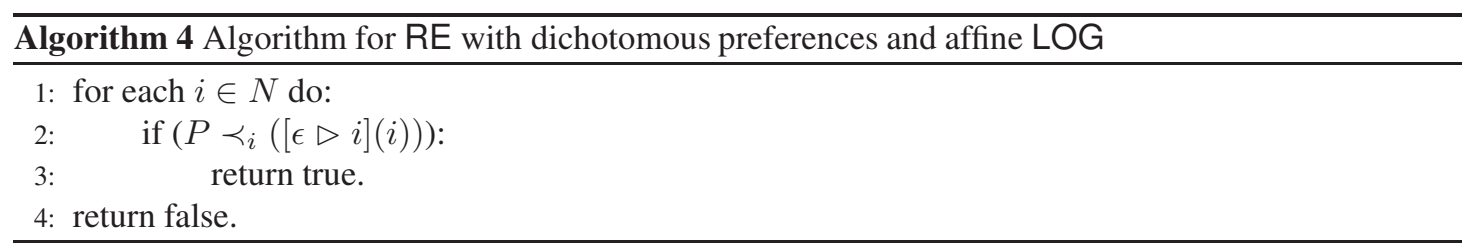

The algorithm is correct. Indeed, by Lemma 17 $P$ is eliminable in $G$ iff there is $i \in N$ where $($ out $(P)) \notin N E\left(G^{[\epsilon \triangleright i]}\right)$. By Lemma[13, we know that $(\operatorname{out}(P)) \notin N E\left(G^{[\epsilon \triangleright i]}\right)$ iff $P \prec_{i}([\epsilon \triangleright i](i))$. Notice that the test of line 2 is equivalent to $P \forall \gamma_{i}$ and $[\epsilon \triangleright i](i) \vdash \gamma_{i}$. Thus, it can be simulated by a deterministic oracle Turing machine in polynomial time with at most $2 n$ non-adaptive queries to an oracle for the problem of sequent provability. When the problem of sequent provability in LOG is in NP it yields a complexity of $P^{N P \|}$. When it is in PTIME (resp., PSPACE), it yields a complexity of PTIME (resp., PSPACE).

\subsubsection{Hardness}

The linear and affine cases both use the same proof strategy which we present at once.

Proposition 20. RE is as hard as the problem of checking sequent non-provability in LOG.

Proof. Let $\Gamma \vdash \delta$ be an arbitrary intuitionistic sequent. Let $\varphi=\Gamma^{*} \multimap \delta$. (Remember that $\Gamma^{*}=$ $\bigotimes_{A \in \Gamma} A$.) Let $G^{\epsilon}=(\{1,2\}, \varphi, \mathbf{1}, \emptyset,\{\varphi\})$ be an individual resource game. So, we have $\epsilon_{1}=\emptyset$ and $\epsilon_{2}=\{\varphi\}$. There is only one other distinct redistribution $\epsilon^{\prime}$ of $\epsilon$ where $\epsilon_{1}^{\prime}=\{\varphi\}$ and $\epsilon_{2}^{\prime}=\emptyset$. It is the case that redis $(\epsilon)=\left\{\epsilon, \epsilon^{\prime}\right\}$. Let $G^{\epsilon^{\prime}}=(\{1,2\}, \varphi, \mathbf{1},\{\varphi\}, \emptyset)$ be the individual resource game resulting from the redistribution $\epsilon^{\prime}$. Both games are represented on Figure 3

We show that both in the case of linear and of affine logics, we have $\Gamma \forall \delta$ iff $(\emptyset, \emptyset)$ is rationally eliminable in $G^{\epsilon}$. 


\begin{tabular}{|c|cc|}
\hline 12 & $\emptyset$ & $\{\varphi\}$ \\
\hline$\emptyset$ & $\emptyset$ & $\{\varphi\}$ \\
\hline
\end{tabular}

(a) $G^{\epsilon}$.

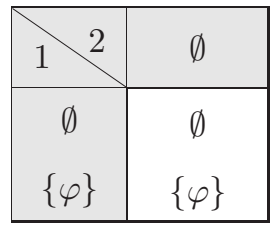

(b) $G^{\epsilon^{\prime}}$.

Figure 3: Games $G^{\epsilon}$ and $G^{\epsilon^{\prime}}$. The profile $(\emptyset, \emptyset)$ is a Nash equilibrium in $G^{\epsilon}$. The profile $(\emptyset, \emptyset)$ is a Nash equilibrium in $G^{\epsilon^{\prime}}$ iff $\Gamma \vdash \delta$. (The profile $(\{\varphi\}, \emptyset)$ is a Nash equilibrium in $G^{\epsilon^{\prime}}$. Depending on whether $\Gamma \vdash \delta$ and whether LOG is linear or affine, $(\emptyset,\{\varphi\})$ may or may not be Nash equilibria in $G^{\epsilon}$. This is inconsequential for the reduction in the proof of Proposition 20]

We first show that

$$
\Gamma \vdash \delta \text { iff } \emptyset \vdash \varphi .
$$

From left to right, suppose $\Gamma \vdash \delta$. By applying $(\otimes \mathrm{L})$ enough times we obtain $\Gamma^{*} \vdash \delta$. Then we obtain $\emptyset \vdash \Gamma^{*} \multimap \delta$ using $(\multimap \mathrm{R})$. From right to left, suppose $\emptyset \vdash \Gamma^{*} \multimap \delta$. With (ax) and $\otimes \mathrm{R}$ we can show $\Gamma \vdash \Gamma^{*}$. Using $\otimes \mathrm{R}$ on the sequents $\Gamma \vdash \Gamma^{*}$ and $\emptyset \vdash \Gamma^{*} \multimap \delta$ we obtain

$$
\Gamma \vdash \Gamma^{*} \otimes \Gamma^{*} \multimap \delta
$$

Without assumption we can also show

$$
\Gamma^{*} \otimes \Gamma^{*} \multimap \delta \vdash \delta
$$

using the rules $(\mathrm{ax}),(\multimap \mathrm{L})$, and $(\otimes \mathrm{L})$. We conclude that $\Gamma \vdash \delta$ using (cut) on the sequents 2 and 3 .

We can proceed. Suppose $\Gamma \nvdash \delta$. We show that $(\emptyset, \emptyset)$ is not a Nash equilibrium in $G^{\epsilon^{\prime}}$. Since $\Gamma \nvdash \delta$, we also have $\emptyset \nvdash \varphi$ (by Equation 1). On the other hand, using (ax), we have $\{\varphi\} \vdash \varphi$. So in the profile $(\emptyset, \emptyset)$, Player 1 has an incentive to deviate to the profile $(\{\varphi\}, \emptyset)$. So $(\emptyset, \emptyset)$ is not a Nash equilibrium in $G^{\epsilon^{\prime}}$.

Suppose $\Gamma \vdash \delta$. We show that $(\emptyset, \emptyset)$ is a Nash equilibrium both in $G^{\epsilon}$ and in $G^{\epsilon^{\prime}}$.

In $G^{\epsilon}$. We have $\emptyset \vdash 1$ from $\mathbf{1 R}$, so Player 2 has no incentive to deviate from the profile $(\emptyset, \emptyset)$ in $G^{\epsilon}$. Moreover, Player 1 is dummy in $G^{\epsilon}$. So $(\emptyset, \emptyset)$ is a Nash equilibrium in $G^{\epsilon}$.

In $G^{\epsilon^{\prime}}$. Since $\Gamma \vdash \delta$, we also have $\emptyset \vdash \varphi$ (by Equation 1), so Player 1 has no incentive to deviate from the profile $(\emptyset, \emptyset)$ in $G^{\epsilon^{\prime}}$. Moreover, Player 2 is dummy in $G^{\epsilon^{\prime}}$. So $(\emptyset, \emptyset)$ is a Nash equilibrium in $G^{\epsilon^{\prime}}$.

\subsection{Construction}

For elimination, Lemma 17 provided a remarkable necessary and sufficient condition for the rational eliminability of a profile. For the rational constructibility of a profile, we can only indicatively provide sufficient conditions. Let $G=\left(N, \gamma_{1}, \ldots, \gamma_{n}, \epsilon_{1}, \ldots, \epsilon_{n}\right)$ be an IRG, and let $P \in \operatorname{ch}(G)$ be a profile in $G$. If there is a player $i \in N$ such that out $(P) \vdash \gamma_{i}$, then $P$ can be rationally constructed by redistributing all the resources to Player $i$. Also, if there is a player $i \in N$ such that $\biguplus_{k \in N} \epsilon_{k} \forall \gamma_{i} \otimes \top$, then $P$ can be rationally constructed by redistributing all the resources to Player $i$.

We tackle the complexity of RATIONAL CONSTRUCTION with dichotomous preferences. 


\subsubsection{Hardness}

We prove a lower bound of the problem RC in presence of dichotomous preferences.

Proposition 21. RC is as hard as the problem of checking sequent provability in LOG.

Proof. Let $\varphi=\Gamma^{*} \multimap \delta$ and $G=\left(\{1\}, \varphi, \epsilon_{1}=\{\varphi\}\right)$. We can see that $(\emptyset) \in N E(G)$ iff $\emptyset \vdash \varphi$, that is $\Gamma \vdash \delta$. As redis $(\epsilon)=\{\epsilon\}$, we conclude that: for every sequent $\Gamma \vdash \delta,(\emptyset)$ is rationally constructible in $G$ iff $\Gamma \vdash \delta$ is provable.

\subsubsection{Algorithms}

Let $G^{\epsilon}$ be an individual resource game, and let $P \in \operatorname{ch}\left(G^{\epsilon}\right)$. To decide whether the profile $P$ can be rationally constructed we can use Algorithm 5. This algorithm will serve for all cases of rational construction in this paper.

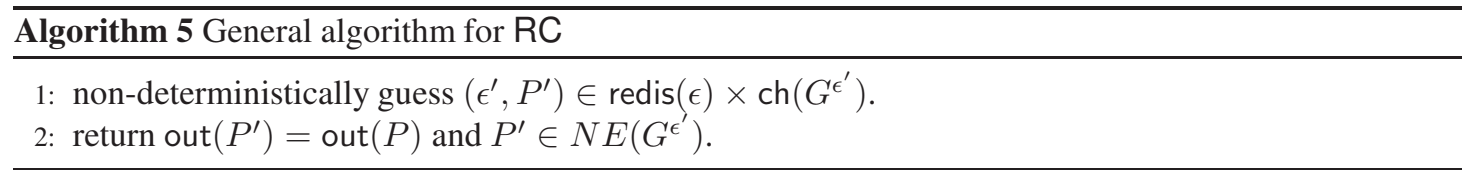

The algorithmic analysis is rather simple: we use the problem NE as a blackbox, for which complexity upper bounds have been established in Proposition 12 and Proposition 15

Proposition 22. When LOG is in PTIME, RC is in $\Sigma_{2}^{\mathrm{p}}$. When LOG is in NP, RC is in $\Sigma_{3}^{\mathrm{p}}$. When LOG is in PSPACE, RC is in PSPACE.

Proof. When LOG is in PTIME, from Proposition 12, we know that the test of line 2 is in coNP. So RC is in NPcoNP $=\Sigma_{2}^{\mathrm{p}}$. Similarly, when LOG is in NP, from Proposition 12, we know that the test of line 2 is in $\Pi_{2}^{\mathrm{p}}$. So $R C$ is in $N P_{2}^{\mathrm{p}}=\Sigma_{3}^{\mathrm{p}}$. The case for $L O G$ in PSPACE is analogous.

Again, an affine LOG seems to bring some relative algorithmic ease.

Proposition 23. If LOG is affine, when provability in LOG is in PTIME, then RC is in NP. When LOG is in NP, RC is in $\Sigma_{2}^{\mathrm{p}}$. When LOG is in PSPACE, RC is in PSPACE.

Proof. The proof is similar to the one of Proposition 22, using the result of Proposition [15 and, for the case of NP the fact that NPP $P^{N P \|} \subseteq N P^{\Delta_{2}^{p}}=\Sigma_{2}^{p}$.

\section{Parsimonious preferences}

Weakening $(W)$ is sometimes a desirable property of LOG and of our preferences of resources. However, it has the untoward consequence of incentivizing players to spend all their resources in individual resource games with dichotomous preferences. This is well exemplified for instance by Proposition 14.

We can teach our players parsimony by attaching to them finer preferences that take into account the realization of their objective, but also the optimality of their contribution.

In an individual resource game $G=\left(N, \gamma_{1}, \ldots, \gamma_{n}, \epsilon_{1}, \ldots, \epsilon_{n}\right)$, we now say that player $i \in N$ (parsimoniously) strongly prefers $P \in \operatorname{ch}(G)$ over $Q \in \operatorname{ch}(G)$ (noted $Q \prec_{i} P$ ) iff one of the following conditions is satisfied:

1. not out $(P) \vdash \gamma_{i}$ and not out $(Q) \vdash \gamma_{i}$ and $P_{i} \subset Q_{i}$;

2. out $(P) \vdash \gamma_{i}$ and not out $(Q) \vdash \gamma_{i}$; 
3. out $(P) \vdash \gamma_{i}$ and out $(Q) \vdash \gamma_{i}$ and $P_{i} \subset Q_{i}$.

Similar preferences have been called pseudo-dichotomous in the literature.

We recognise that the second condition corresponds to profile $P$ being dichotomously strongly preferred by Player $i$ to profile $Q$. The following proposition is a simple consequence.

Lemma 24. If Player $i$ dichotomously strongly prefers $P$ over $Q$ then Player $i$ parsimoniously strongly prefers $P$ over $Q$.

This has another immediate consequence on Nash equilibria.

Lemma 25. If a profile $P$ is a Nash equilibrium in presence of parsimonious preferences, then $P$ is a Nash equilibrium in presence of dichotomous preferences.

Proof. Let $\prec_{i}^{d}$ (resp., $\prec_{i}^{p}$ ) denote Player $i$ 's parsimonious (resp., dichotomous) preferences; Let $N E_{d}(G)$ (resp., $N E_{p}(G)$ ) denote the set of Nash equilibria in $G$ when considering dichotomous (resp., parsimonious) preferences. Now suppose that $P \in N E_{p}(G)$. That is, for every $i \in N$ and for every $C_{i} \in \operatorname{ch}_{i}(G)$ we have not $P \prec_{i}^{p}\left(C_{i}, P_{-i}\right)$, and by Lemma 24, we have not $P \prec_{i}^{d}\left(C_{i}, P_{-i}\right)$. So $P \in N E_{d}(G)$.

Lemma 25 indicates that every Nash equilibrium in presence of parsimonious preferences is also a Nash equilibrium in presence of dichotomous preferences. The next proposition, which will help us later to prove some hardness result, says that the other way around holds when the profile is the one where every player plays the empty set of resources.

Lemma 26. The profile $(\emptyset, \ldots, \emptyset)$ is a Nash equilibrium in presence of parsimonious preferences iff it is a Nash equilibrium in presence of dichotomous preferences.

Proof. Left to right is a consequence of Lemma 25. For right to left, assume $(\emptyset, \ldots, \emptyset)$ is in $N E_{d}(G)$. With parsimonious preferences, the only incentive to deviate from a Nash equilibrium in presence of dichotomous preferences, would be to play a smaller multiset of resources. This is impossible in $(\emptyset, \ldots, \emptyset)$.

We now address the complexity of the decision problem of deciding whether a player parsimoniously strongly prefers a profile over another profile.

Proposition 27. Let $G=\left(N, \gamma_{1}, \ldots, \gamma_{n}, \epsilon_{1}, \ldots, \epsilon_{n}\right)$ be an individual resource game. Let also $P \in$ $\operatorname{ch}(G)$ and $Q \in \operatorname{ch}(G)$ be two profiles, and $i \in N$ be a player. The problem of deciding whether $Q \prec_{i} P$ is: in PTIME when provability in LOG is in PTIME. It is in $\mathrm{P}^{\mathrm{NP}} \|[2]$ when provability in LOG is NPcomplete. It is in PSPACE when provability in LOG is PSPACE-complete.

Proof. First, we can evaluate $P_{i} \subseteq Q_{i}$ efficiently. We store the result in the Boolean variable $v_{\subseteq}$.

We can then perform two non-adaptive queries to an oracle to solve sequent validity in LOG on out $(P) \vdash \gamma_{i}$ and on out $(Q) \vdash \gamma_{i}$, and store the results in the Boolean variables $v_{P}$ and $v_{Q}$ respectively. The formula $\left(\left(\neg v_{p} \wedge \neg v_{q} \wedge v_{\subseteq}\right) \vee\left(v_{p} \wedge \neg v_{q}\right) \vee\left(v_{p} \wedge v_{q} \wedge v_{\subseteq}\right)\right)$ is true iff $Q \prec_{i} P$.

This yields a correct algorithm for deciding $Q \prec_{i} P$ in PTIME when LOG is in PTIME, in PNP\|[2] when LOG is in NP, and in PSPACE when LOG is in PSPACE.

To compare the complexity of dichotomous and parsimonious preferences, remember from Proposition 10 that when LOG is in NP, the same problem for dichotomous preferences is in $\mathrm{BH}_{2}$. From [21] we know that $\mathrm{P}^{\mathrm{NP} \|[1]} \subseteq \mathrm{BH}_{2} \subseteq \mathrm{P}^{\mathrm{NP} \|[2]}$. It is not known whether these inclusions are strict. 


\subsection{Illustration of redistribution and parsimony}

Consider again the individual resource game of Section 4.2 (Unless stated otherwise, suppose we are in the affine case.) With parsimonious preferences, we have $N E(G)=\{(\emptyset, \emptyset)\}$. The profile $(\{A\},\{B\})$ is not a Nash equilibrium as it was with dichotomous preferences. It would be more desirable from a social welfare point of view than any other outcome (it satisfies both players), but the players would nonetheless not be individually rational by choosing it. They have indeed no bearing upon the outcome that satisfies them and thus are rational in withholding their resources.

Nonetheless, like in the case of dichotomous preference, we can effectively eliminate the current Nash equilibrium in $G^{\epsilon}$ and construct the Nash equilibrium yielding $\{A, B\}$ by redistributing the resources present in $G^{\epsilon}$ so as to obtain $G^{\epsilon^{\prime}}=\left(\{1,2\}, \gamma_{1}=B, \gamma_{2}=A,\{B\},\{A\}\right)$. The only Nash equilibrium is now $(\{B\},\{A\})$.

Unlike dichotomous preferences, parsimonious preferences do not ensure the existence of a Nash equilibrium in the affine case. Consider the individual resource game $H^{\epsilon}=\left(\{1,2\}, \gamma_{1}=A, \gamma_{2}=\right.$ $A \otimes A,\{A\},\{A\})$. There are two players. The game $H^{\epsilon}$ can be represented as on Figure 4

\begin{tabular}{|c|c|c|}
\hline $1 \quad 2$ & $\emptyset$ & $\{A\}$ \\
\hline$\emptyset$ & $\emptyset$ & $\{A\}: \gamma_{1}$ \\
\hline$\{A\}$ & $\{A\}: \gamma_{1}$ & $\{A, A\}: \gamma_{1}, \gamma_{2}$ \\
\hline
\end{tabular}

Figure 4: The game $H^{\epsilon}$. There is no Nash equilibrium under parsimonious preferences.

The game $H^{\epsilon}$ has no Nash equilibrium: At $(\emptyset, \emptyset)$, Player 1 does not realize her objective, but she can deviate and play $\{A\}$ to satisfy it. At $(\{A\}, \emptyset)$, Player 2 has an incentive to deviate and play $\{A\}$ to realize her objective. At $(\{A\},\{A\})$ Player 1 has an incentive to deviate and play $\emptyset$. (In the affine case this is because she can still satisfy her objective by contributing less. In the linear case, this is because she can satisfy her objective while she does not before deviating.) At $(\emptyset,\{A\})$, Player 2 does not satisfy her objective and thus has an incentive to deviate to play $\emptyset$.

However, we can construct the Nash equilibrium yielding $\{A, A\}$. Let $\epsilon^{\prime}$ be the redistribution of $\epsilon$ such that $\epsilon^{\prime}(2)=\{A, A\}$ and $\epsilon^{\prime}(1)=\emptyset$. We obtain the game depicted on Figure 5

\begin{tabular}{|c|c|c|c|}
\hline 1 & $\emptyset$ & $\{A\}$ & $\{A, A\}$ \\
\hline$\emptyset$ & $\emptyset$ & $\{A\}: \gamma_{1}$ & $\{A, A\} \boldsymbol{\square}: \gamma_{1}, \gamma_{2}$ \\
\hline
\end{tabular}

Figure 5: The game $H^{\epsilon^{\prime}}$. The symbol $\mathbf{\square}$ denotes a Nash equilibrium.

In $H^{\epsilon^{\prime}}$, by assigning all the resources to Player 2, the profile $(\emptyset,\{A, A\})$ is a Nash equilibrium and the only one. In affine logics, both players satisfy their objectives, but only Player 2 does when the logic is linear.

\subsection{Finding Nash equilibria}

We study the complexity of NASH EQUILIBRIUM with parsimonious preferences. 


\subsubsection{Hardness}

We are now getting used to many-to-one reductions from sequent (non-)provability. It was a fruitful problem in presence of dichotomous preference, and it will remain one in presence of parsimonious preferences. We prove a complexity lower bound for the problem of NE in presence of parsimonious preferences.

Proposition 28. The problem NE is as hard as the problem of checking sequent non-provability in LOG, even when there is only one player.

Proof. As before, we consider w.l.o.g. only the intuitionistic sequents of LOG in the following reduction.

Let $\Gamma \vdash \delta$ be an intuitionistic sequent of LOG. We define $\varphi=\Gamma^{*} \multimap \delta$. We can construct the individual resource game $G$ such that $G=(\{1\}, \varphi,\{\varphi\})$. In $G$, Player 1 has exactly two choices: $\operatorname{ch}_{i}(G)=\{\emptyset,\{\varphi\}\}$.

We show that $\Gamma \vdash \delta$ iff $\varphi \notin N E(G)$.

Suppose $(\{\varphi\}) \notin N E(G)$. So $(\{\varphi\}) \prec_{1}(\emptyset)$. Since by (ax) $\varphi \vdash \varphi$ (the profile $(\{\varphi\})$ satisfies Player 1's objectives) and $\emptyset \subset\{\varphi\}$ (Player 1's contribution is strictly less in the profile $(\emptyset)$ than it is in $(\{\varphi\}))$, it must be that $\emptyset \vdash \varphi$. We infer $\Gamma \vdash \delta$, as we did in part of the proof of Proposition 20 .

Suppose $\Gamma \vdash \delta$. We obtain $\Gamma^{*} \vdash \delta$ by using $(\otimes \mathrm{L})$ enough times, and we deduce $\vdash \varphi$ with $(\multimap \mathrm{R})$. We thus have $\emptyset \vdash \varphi$ and $\emptyset \subset\{\varphi\}$. So $(\{\varphi\}) \prec_{1}(\emptyset)$ and $(\{\varphi\}) \notin N E(G)$.

\subsubsection{Algorithms}

In the individual resource game $G=\left(N, \gamma_{1}, \ldots, \gamma_{n}, \epsilon_{1}, \ldots, \epsilon_{n}\right)$, we can use Algorithm 1 to check whether a profile $P \notin N E(G)$, even for parsimonious preferences. We have a result analogous to Proposition 12 for parsimonious preferences.

Proposition 29. If the problem of sequent provability in LOG is in PTIME then NE is in coNP. If the problem of sequent provability in LOG is in NP then NE is in $\Pi_{2}^{\mathrm{p}}$. If the problem of sequent provability in LOG is in PSPACE then NE is in PSPACE.

Proof. We use Proposition 27 and, for the case of NP, the fact that coNP $\mathrm{P}^{\mathrm{NP} \mid\left[{ }^{[2]}[1]\right.} \subseteq \operatorname{coNP}^{\mathrm{P}^{\mathrm{NP}}}=$ $\operatorname{coNP}_{2}^{\mathrm{p}}=\operatorname{co}_{2}^{\mathrm{p}}=\Pi_{2}^{\mathrm{p}}$.

When LOG is affine, we can do better than using Algorithm 1, We first state a technical lemma which is analogous to Lemma 13 .

Lemma 30. Let $G=\left(N, \gamma_{1}, \ldots, \gamma_{n}, \epsilon_{1}, \ldots, \epsilon_{n}\right)$ be an individual resource game. When LOG is affine, $P \notin N E(G)$ iff $\exists i \in N$ : s.t. either:

1. $\operatorname{out}(P) \forall \gamma_{i}$ and $P_{i} \neq \emptyset$;

2. $\operatorname{out}(P) \forall \gamma_{i}$ and $\operatorname{out}\left(\left(P_{-i}, \epsilon_{i}\right)\right) \vdash \gamma_{i}$;

3. out $(P) \vdash \gamma_{i}$ and $\exists A \in P_{i}$ : out $\left(\left(P_{-i}, P_{i} \backslash\{A\}\right)\right) \vdash \gamma_{i}$.

Proof. Right to left is immediate. From left to right, suppose $P \notin N E(G)$. So there exists $i \in N$ and $C_{i} \in \operatorname{ch}_{i}(G)$ such that $P \prec_{i}\left(P_{-i}, C_{i}\right)$. There are three cases to consider:

1. not out $\left(\left(P_{-i}, C_{i}\right)\right) \vdash \gamma_{i}$ and not out $(P) \vdash \gamma_{i}$ and $C_{i} \subset P_{i}$;

2. out $\left(\left(P_{-i}, C_{i}\right)\right) \vdash \gamma_{i}$ and not out $(P) \vdash \gamma_{i}$;

3. out $\left(\left(P_{-i}, C_{i}\right)\right) \vdash \gamma_{i}$ and out $(P) \vdash \gamma_{i}$ and $C_{i} \subset P_{i}$. 
Suppose (1) is the case. It implies that there is $C_{i} \subset P_{i}$ and thus that $P_{i} \neq \emptyset$. Suppose (2) is the case. We essentially use the same argument as the one used in the proof of Lemma 13. We have out $\left(\left(P_{-i}, C_{i}\right)\right) \vdash$ $\gamma_{i}$. By applying weakening $\left(\left|\epsilon_{i}\right|-\left|C_{i}\right|\right)$ times, we easily obtain that out $\left(\left(P_{-i}, \epsilon_{i}\right)\right) \vdash \gamma_{i}$. Suppose (3) is the case. We thus have out $\left(\left(P_{-i}, C_{i}\right)\right) \vdash \gamma_{i}$ with $C_{i} \subset P_{i}$. Take a formula $A \in P_{i} \backslash C_{i}$. Then, by applying weakening $\left(\left|P_{i}\right|-\left|C_{i}\right|-1\right)$ times, we easily obtain that out $\left(\left(P_{-i}, P_{i} \backslash\{A\}\right)\right) \vdash \gamma_{i}$.

Algorithm 6 can then be used to check whether $P \in N E(G) 6$

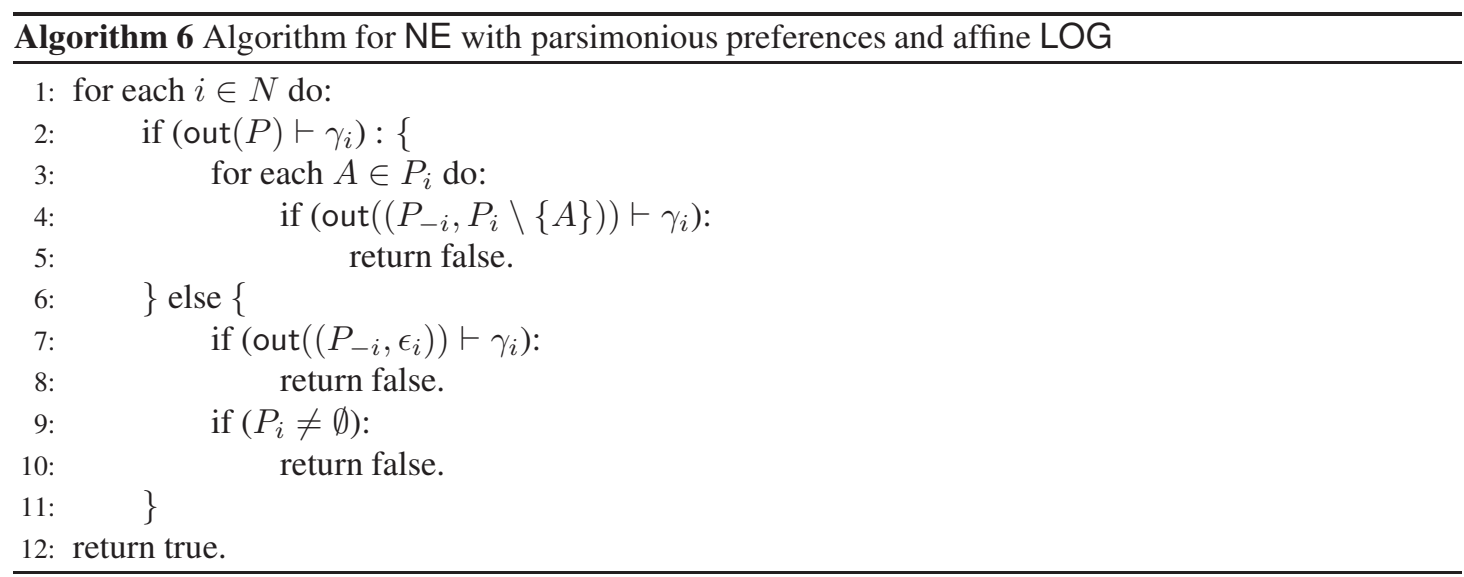

Proposition 31. When LOG is affine, if the problem of sequent provability in LOG is in PTIME, then NE is in $\mathrm{PTIME}$. If the problem of sequent provability in LOG is in NP, then $\mathrm{NE}$ is in $\mathrm{P} \mathrm{NP} \|$. If the problem of sequent provability in LOG is in PSPACE, then NE is in PSPACE.

Proof. Lemma 30 justifies the correctness of Algorithm 6. The algorithm can be simulated by a deterministic oracle Turing machine in polynomial time with less than $\Sigma_{i \in N}\left(1+\left|P_{i}\right|\right)$ non-adaptive queries to an oracle for sequent provability in LOG. When the complexity of sequent provability in LOG is in NP it yields a complexity of $\mathrm{P}^{\mathrm{NP} \|}$.

\subsection{Elimination}

We study the complexity of RATIONAL ELIMINATION with parsimonious preferences.

\subsubsection{Algorithms}

Lemma 17 also holds for parsimonious preferences. It is easy to see that the proof carries over.

Algorithm 3 can still be used in the case of parsimonious preferences because Lemma 17 is still granted. We thus have the analog to Proposition 18 for parsimonious preferences.

Proposition 32. When LOG is linear, RE is in NP when sequent provability in LOG is in PTIME, in $\Sigma_{2}^{\mathrm{p}}$ when LOG is in NP, and in PSPACE when LOG is in PSPACE.

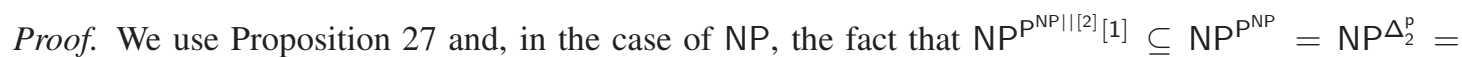
$\Sigma_{2}^{\mathrm{p}}$.

Let $G=\left(N, \gamma_{1}, \ldots, \gamma_{n}, \epsilon_{1}, \ldots, \epsilon_{n}\right)$ be an individual resource game and let $P \in \operatorname{ch}(G)$ be a profile. We can use Algorithm 7 to check whether a profile $P \in \operatorname{ch}(G)$ is rationally eliminable.

\footnotetext{
${ }^{6}$ Algorithm 6 corrects an omission in $\left[42\right.$ Algo. 5] by adding "if $\left(P_{i} \neq \emptyset\right)$ : return false" lines 9 and 10.
} 


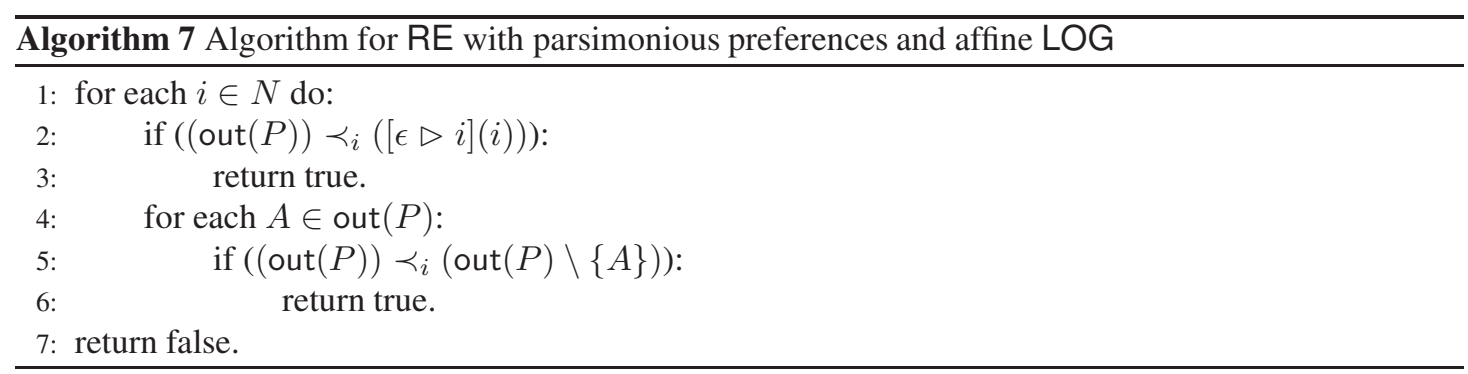

Proposition 33. When LOG is affine, RE is in PTIME when provability in LOG is in PTIME. It is in $\mathrm{P}^{\mathrm{NP} \|}$ when LOG is in NP. It is in PSPACE when LOG is in PSPACE.

Proof. Lemma 17 which still holds with parsimonious preferences ensures that it is enough to consider the redistributions $[\epsilon \triangleright i]$ for some player $i$. Algorithm [7, then checks for each of these redistributions whether Player $i$ has an incentive to deviate in the game $G^{[\epsilon \triangleright i]}$ from the profile (out $\left.(P)\right) \in \operatorname{ch}\left(G^{[\epsilon \triangleright i]}\right)$ to any one of $([\epsilon \triangleright i](i)) \in \operatorname{ch}\left(G^{[\epsilon \triangleright i]}\right)$ and (out $\left.(P) \backslash\{A\}\right) \in \operatorname{ch}\left(G^{[\epsilon \triangleright i]}\right)$ for some $A \in \operatorname{out}(P)$. It is weakening $(W)$ that justifies that it is enough to consider these profiles, because $X \forall \gamma_{i}$ implies $Y \nvdash \gamma_{i}$ for any couple of multisets $Y \subseteq X$. The correctness of Algorithm 7 follows.

The tests of line 2 and line 5 only involve the following instances of the sequent provability decision problem: $(\operatorname{out}(P)) \vdash \gamma_{i}$ and $([\epsilon \triangleright i](i)) \vdash \gamma_{i}$ for very Player $i \in N$, and $(\operatorname{out}(P) \backslash\{A\}) \vdash \gamma_{i}$, for every Player $i \in N$ and every formula $A \in$ out $(P)$. The algorithm can thus be simulated by a deterministic oracle Turing machine in polynomial time with at most $|N|(\mid$ out $(P) \mid+2)$ non-adaptive calls to an oracle for sequent provability.

\subsubsection{Hardness}

After Lemma 26 and the proof of Proposition 20, the following proposition does not come as a surprise.

Proposition 34. RE is as hard as the problem of checking sequent non-provability in LOG.

Proof. Let $\Gamma \vdash \delta$ be an arbitrary intuitionistic sequent. We construct the same game as in the proof of Proposition 20. Let $\varphi=\Gamma^{*} \multimap \delta$. Let $G^{\epsilon}=(\{1,2\}, \varphi, \mathbf{1}, \emptyset,\{\varphi\})$.

In the proof of Proposition 20, we showed that, in presence of dichotomous preferences, both in the case of linear and of affine logics, we have $\Gamma \nvdash \delta$ iff $(\emptyset, \emptyset)$ is rationally eliminable in $G^{\epsilon}$.

Now with Lemma 26, we know that $(\emptyset, \emptyset)$ is a Nash equilibrium in presence of dichotomous preferences iff it is a Nash equilibrium in presence of parsimonious preferences (both in $G^{\epsilon}$ and $G^{\epsilon^{\prime}}$, and no matter if LOG is linear or affine, or if $\Gamma \vdash \delta$ or $\Gamma \forall \delta$ ).

Hence, we have $\Gamma \forall \delta$ iff $(\emptyset, \emptyset)$ is rationally eliminable in $G^{\epsilon}$, also in presence of parsimonious preferences.

\subsection{Construction}

Finally, we tackle the complexity of RATIONAL CONSTRUCTION with parsimonious preferences.

\subsubsection{Hardness}

We establish a complexity lower bound for the problem of $\mathrm{RC}$ in presence of parsimonious preferences.

Proposition 35. RC is as hard as the problem of checking sequent non-provability in LOG.

Proof. Consider the games in the proof of Proposition 34 We can see that both for linear and affine logics we have that $\Gamma \nvdash \delta$ iff $(\{\varphi\}, \emptyset)$ can be rationally constructed in $G^{\epsilon^{\prime}}$. 


\subsubsection{Algorithms}

Our algorithmic analysis is very similar to the analysis we made when the preferences are dichotomous in Section 4.3.2 Let $G^{\epsilon}$ be an individual resource game and $P \in \operatorname{ch}\left(G^{\epsilon}\right)$. To decide whether $P$ can be rationally constructed we can reuse Algorithm 5

Again, we use the problem NE as a blackbox, for which complexity upper bounds have been established in Proposition 29 and Proposition 31

Proposition 36. When sequent provability in LOG is in PTIME, RC is in $\Sigma_{2}^{\mathrm{p}}$. When $\mathrm{LOG}$ is in NP, RC is in $\Sigma_{3}^{\mathrm{p}}$. When LOG is in PSPACE, RC is in PSPACE.

Proof. The proof is similar to the one of Proposition 22, using the result of Proposition 29

The next proposition also comes without surprise.

Proposition 37. If LOG is affine, when LOG is in PTIME, RC is in NP. When LOG is in NP, RC is in $\Sigma_{2}^{\mathrm{p}}$. When $\mathrm{LOG}$ is in PSPACE, RC is in PSPACE.

Proof. The proof is similar to the one of Proposition 23, using the result of Proposition 31

\section{Examples}

We present more thorough examples. They involve several resources and objectives that are modeled with a variety of logical operands. We take the opportunity to present fully the important formal proofs of the realized objectives.

We start with a toy example, simple but rich enough, upon which we can demonstrate all the frameworks and problems addressed in the paper.

Then, we formally study the divorce arbitration scenario of Example 2 as well as a three-player variant of the scenario of interconnected economies from Example 1

\subsection{Alan and the fish}

We first introduce the resources involved and how they are built in the logical language.

- Basic resources:

- one mole of dioxygen: $\mathrm{O}_{2}$

- one mole of dihydrogen: $\mathrm{H}_{2}$

- one mole of water: $\mathrm{H}_{2} \mathrm{O}$

- one 'token' of thirst: thirst

- Anti-resources can be captured via the linear negation:

- one thirst quencher: $\sim$ thirst

- Resource transformation processes:

- one process of electrolysis: elec $=\mathrm{H}_{2} \mathrm{O} \otimes \mathrm{H}_{2} \mathrm{O} \multimap \mathrm{H}_{2} \otimes \mathrm{H}_{2} \otimes \mathrm{O}_{2}$

- one process of drinking water: drink $=\mathrm{H}_{2} \mathrm{O} \multimap \sim$ thirst 
Game definition. Let $G_{a f}^{\epsilon}=\left(\{a, f\}, \gamma_{a}, \gamma_{f}, \epsilon_{a}, \epsilon_{f}\right)$ be the individual resource game with two players, Alan $a$ and the Fish $f$. The fish wants one mole of dioxygen: $\gamma_{f}=\mathrm{O}_{2}$. Alan wants one mole of dioxygen for his fish and wants to quench his thirst: $\gamma_{a}=\mathrm{O}_{2} \otimes \sim$ thirst.

In the game $G_{a f}^{\epsilon}$, Alan is endowed with $\epsilon_{a}=\{$ drink, elec $\}$. He can drink once and can electrolyse water once. The fish is endowed with three tokens of water $\epsilon_{f}=\left\{\mathrm{H}_{2} \mathrm{O}, \mathrm{H}_{2} \mathrm{O}, \mathrm{H}_{2} \mathrm{O}\right\}$.

We suppose that LOG is affine. For this example, we will consider both cases of dichotomous and parsimonious preferences.

As we did before, we will represent a Nash equilibrium under dichotomous preferences with the symbol $\square$, and under parsimonious preferences with the symbol $\mathbf{\square}$. By Lemma25, the latter implies the former. Then, when a profile is a Nash equilibrium under both dichotomous and parsimonious preferences we will use the symbol $\mathbf{\square}$. The game $G_{a f}^{\epsilon}$ and the realized objectives can be depicted as on Figure 6 ,

\begin{tabular}{|c|cccc|}
\hline$a$ & $\emptyset$ & $\left\{\mathrm{H}_{2} \mathrm{O}\right\}$ & $\left\{\mathrm{H}_{2} \mathrm{O}, \mathrm{H}_{2} \mathrm{O}\right\}$ & $\left\{\mathrm{H}_{2} \mathrm{O}, \mathrm{H}_{2} \mathrm{O}, \mathrm{H}_{2} \mathrm{O}\right\}$ \\
\hline$\emptyset$ & $\emptyset$ & $\left\{\mathrm{H}_{2} \mathrm{O}\right\}$ & $\left\{\mathrm{H}_{2} \mathrm{O}, \mathrm{H}_{2} \mathrm{O}\right\} \square$ & $\left\{\mathrm{H}_{2} \mathrm{O}, \mathrm{H}_{2} \mathrm{O}, \mathrm{H}_{2} \mathrm{O}\right\}$ \\
$\{$ drink & $\{$ drink $\square$ & $\left\{\right.$ drink, $\left.\mathrm{H}_{2} \mathrm{O}\right\}$ & $\left\{\right.$ drink, $\left.\mathrm{H}_{2} \mathrm{O}, \mathrm{H}_{2} \mathrm{O}\right\}$ & $\left\{\right.$ drink, $\left.\mathrm{H}_{2} \mathrm{O}, \mathrm{H}_{2} \mathrm{O}, \mathrm{H}_{2} \mathrm{O}\right\}$ \\
$\{$ elec $\}$ & $\{$ elec $\}$ & $\left\{\right.$ elec, $\left.\mathrm{H}_{2} \mathrm{O}\right\}$ & $\left\{\right.$ elec, $\left.\mathrm{H}_{2} \mathrm{O}, \mathrm{H}_{2} \mathrm{O}\right\} \square: \gamma_{f}$ & $\left\{\right.$ elec, $\left.\mathrm{H}_{2} \mathrm{O}, \mathrm{H}_{2} \mathrm{O}, \mathrm{H}_{2} \mathrm{O}\right\}: \gamma_{f}$ \\
$\{$ drink, elec $\}$ & $\{$ drink, elec $\}$ & $\left\{\right.$ drink, elec, $\left.\mathrm{H}_{2} \mathrm{O}\right\}$ & $\left\{\right.$ drink, elec, $\left.\mathrm{H}_{2} \mathrm{O}, \mathrm{H}_{2} \mathrm{O}\right\} \square: \gamma_{f}$ & $\left\{\right.$ drink, elec, $\left.\mathrm{H}_{2} \mathrm{O}, \mathrm{H}_{2} \mathrm{O}, \mathrm{H}_{2} \mathrm{O}\right\} \square: \gamma_{a}, \gamma_{f}$ \\
\hline
\end{tabular}

Figure 6: The game $G_{a f}^{\epsilon}$. Alan plays rows, and the fish plays columns. LOG is affine. The symbol $\square$ marks the Nash equilibria under dichotomous preferences. The symbol $\mathbf{\square}$ marks the profiles that are also Nash equilibria under both dichotomous and parsimonious preferences.

Appendix Cprovides the detailed proofs of the realized objectives.

Dichotomous preferences: eliminations of bad equilibria. If the preferences are dichotomous, there are plenty Nash equilibria in $G_{a f}^{\epsilon}$. They are: $(\emptyset, \emptyset),\left(\emptyset,\left\{\mathrm{H}_{2} \mathrm{O}\right\}\right),\left(\emptyset,\left\{\mathrm{H}_{2} \mathrm{O}, \mathrm{H}_{2} \mathrm{O}\right\}\right),(\{$ drink $\}, \emptyset),(\{$ drink $\}$, $\left.\left\{\mathrm{H}_{2} \mathrm{O}\right\}\right),\left(\{\right.$ drink $\left.\},\left\{\mathrm{H}_{2} \mathrm{O}, \mathrm{H}_{2} \mathrm{O}\right\}\right),\left(\{\right.$ elec $\left.\},\left\{\mathrm{H}_{2} \mathrm{O}, \mathrm{H}_{2} \mathrm{O}\right\}\right),\left(\{\right.$ drink, elec $\left.\},\left\{\mathrm{H}_{2} \mathrm{O}, \mathrm{H}_{2} \mathrm{O}\right\}\right)$, and (\{drink, elec $\}$, $\left.\left\{\mathrm{H}_{2} \mathrm{O}, \mathrm{H}_{2} \mathrm{O}, \mathrm{H}_{2} \mathrm{O}\right\}\right)$.

However, only the profile ( $\{$ drink, elec $\},\left\{\mathrm{H}_{2} \mathrm{O}, \mathrm{H}_{2} \mathrm{O}, \mathrm{H}_{2} \mathrm{O}\right\}$ ), whose outcome is $\left\{\right.$ drink, $\mathrm{H}_{2} \mathrm{O}, \mathrm{H}_{2} \mathrm{O}, \mathrm{H}_{2} \mathrm{O}$, elec $\}$, satisfies the objectives of both players. It would thus be desirable to eliminate the other profiles. To do so, let $\epsilon^{\prime}$ be the endowment such that $\epsilon_{a}^{\prime}=\left\{\right.$ drink, elec, $\left.\mathrm{H}_{2} \mathrm{O}, \mathrm{H}_{2} \mathrm{O}, \mathrm{H}_{2} \mathrm{O}\right\}$ and $\epsilon_{f}^{\prime}=\emptyset$. The game $G_{a f}^{\epsilon^{\prime}}$ and the realized objectives can be (partially) depicted as on Figure 7

It is readily seen that in $G_{a f}^{\epsilon^{\prime}}$, when preferences are dichotomous, only the profile ( $\left\{\right.$ drink, elec, $\left.\mathrm{H}_{2} \mathrm{O}, \mathrm{H}_{2} \mathrm{O}, \mathrm{H}_{2} \mathrm{O}\right\}, \emptyset$ ) whose outcome is $\left\{\right.$ drink, $\mathrm{H}_{2} \mathrm{O}, \mathrm{H}_{2} \mathrm{O}, \mathrm{H}_{2} \mathrm{O}$, elec $\}$, is a Nash equilibrium.

Parsimonious preferences: construction of a good equilibrium. If the preferences are parsimonious, the profile $(\emptyset, \emptyset)$ is a Nash equilibrium in the game $G_{a f}^{\epsilon}$, and is the only one. One can nonetheless redistribute the resources so as to construct an equilibrium where Alan and the fish both realize their objectives. That is, one can construct the profile ( $\{$ drink, elec $\},\left\{\mathrm{H}_{2} \mathrm{O}, \mathrm{H}_{2} \mathrm{O}, \mathrm{H}_{2} \mathrm{O}\right\}$ ). To do so, let $\epsilon^{\prime \prime}$ be the endowment such that $\epsilon_{a}^{\prime \prime}=\left\{\right.$ drink, $\left.\mathrm{H}_{2} \mathrm{O}, \mathrm{H}_{2} \mathrm{O}, \mathrm{H}_{2} \mathrm{O}\right\}$ and $\epsilon_{f}^{\prime \prime}=\{\mathrm{elec}\}$. The game $G_{a f}^{\epsilon^{\prime \prime}}$ and the realized objectives can be depicted as on Figure 8

When preferences are parsimonious, the profiles $(\emptyset, \emptyset)$ and ( $\left\{\right.$ drink, $\left.\mathrm{H}_{2} \mathrm{O}, \mathrm{H}_{2} \mathrm{O}, \mathrm{H}_{2} \mathrm{O}\right\},\{$ elec $\left.\}\right)$ are Nash equilibria in $G_{a f}^{\epsilon^{\prime \prime}}$ and are the only ones.

Notice that, the redistribution $\epsilon^{\prime}$ would also effectively construct the profile, although at the price of a more draconian redistribution. It would also eliminate $(\emptyset, \emptyset)$. 


\begin{tabular}{|c|c|}
\hline \multicolumn{1}{|c|}{$f$} & $\emptyset$ \\
\hline$\emptyset$ & $\emptyset$ \\
$\{$ drink $\}$ & $\{$ drink $\}$ \\
$\vdots$ & $\vdots$ \\
$\left\{\right.$ elec, $\left.\mathrm{H}_{2} \mathrm{O}, \mathrm{H}_{2} \mathrm{O}\right\}$ & $\left\{\right.$ elec, $\left.\mathrm{H}_{2} \mathrm{O}, \mathrm{H}_{2} \mathrm{O}\right\}: \gamma_{f}$ \\
$\vdots$ & $\vdots$ \\
$\left\{\right.$ elec, $\left.\mathrm{H}_{2} \mathrm{O}, \mathrm{H}_{2} \mathrm{O}, \mathrm{H}_{2} \mathrm{O}\right\}$ & $\left\{\right.$ elec, $\left.\mathrm{H}_{2} \mathrm{O}, \mathrm{H}_{2} \mathrm{O}, \mathrm{H}_{2} \mathrm{O}\right\}: \gamma_{f}$ \\
$\vdots$ & $\vdots$ \\
$\left\{\right.$ drink, elec, $\left.\mathrm{H}_{2} \mathrm{O}, \mathrm{H}_{2} \mathrm{O}\right\}$ & $\left\{\right.$ drink, elec, $\left.\mathrm{H}_{2} \mathrm{O}, \mathrm{H}_{2} \mathrm{O}\right\}: \gamma_{f}$ \\
$\left\{\right.$ drink, elec, $\left.\mathrm{H}_{2} \mathrm{O}, \mathrm{H}_{2} \mathrm{O}, \mathrm{H}_{2} \mathrm{O}\right\}$ & $\left\{\right.$ drink, elec, $\left.\mathrm{H}_{2} \mathrm{O}, \mathrm{H}_{2} \mathrm{O}, \mathrm{H}_{2} \mathrm{O}\right\} \mathbf{\square}: \gamma_{a}, \gamma_{f}$ \\
\hline
\end{tabular}

Figure 7: The game $G_{a f}^{\epsilon^{\prime}}$.

\subsection{Ann and Bernard get a divorce}

We formalize Example 2 We will only consider parsimonious preferences. We also assume that LOG is Affine MLL. We introduce the resources involved in the example.

- the lease agreement: shop

- the resource of flour for a year: flour

- the resource of one year worth of bread: bread

- the bread making equipment is the resource transformation process: flour $\multimap$ bread

Using these as basic resources, we formalize Example 2 as the game $G_{a b}^{\epsilon}$.

Game definition. Let $G_{a b}^{\epsilon}=\left(\{a, b\}, \gamma_{a}, \gamma_{b}, \epsilon_{a}, \epsilon_{b}\right)$ be the individual resource game with two players, Ann $a$ and Bernard $b$. Ann wants enough bread for a year: $\gamma_{a}=$ bread. Bernard wants the lease agreement: $\gamma_{b}=$ shop. In the game $G_{a b}^{\epsilon}$, Ann is endowed with the lease agreement: $\epsilon_{a}=$ \{shop\}. Bernard is endowed with enough flour to make bread for two years, and with the bread making equipment: $\epsilon_{b}=\{$ flour, flour, flour $\multimap$ bread $\}$.

The game $G_{a b}^{\epsilon}$ and the realized objectives can be depicted as on Figure 9 All the formal proofs of the realized objectives are trivial.

An undesirable equilibrium. One can see on Figure 9 that the profiles $(\{f l o u r$, flour $\multimap$ bread $\}$, $\{$ shop $\})$ and (\{flour, flour, flour $\multimap$ bread $\},\{$ shop $\})$ in $\mathrm{ch}_{b} \times \mathrm{ch}_{a}$ would satisfy both Ann and Bernard. However, in both of them, Bernard has an incentive to provide less resources from his endowment, and to deviate to $\emptyset \in \mathrm{ch}_{b}$. In turn, in $(\emptyset,\{$ shop $\}) \in \mathrm{ch}_{b} \times \mathrm{ch}_{a}$, Ann is not satisfied, and so has an incentive to retain her resources as well, deviating to her choice $\emptyset \in \mathrm{ch}_{a}$. The profiles ( $\{$ flour, flour $\multimap$ bread $\}, \emptyset$ ) and $(\{$ flour, flour, flour $\multimap$ bread $\}, \emptyset\})$ in $\mathrm{ch}_{b} \times \mathrm{ch}_{a}$ satisfy Ann's objective but do not satisfy Bernard's. Hence, Bernard has an incentive to deviate to $\emptyset \in \mathrm{ch}_{b}$.

The profile $(\emptyset, \emptyset)$ is the only Nash equilibrium of $G_{a b}^{\epsilon}$, but it satisfies neither Ann's objective, nor Bernard's. On the other hand, the outcome of the profile ( $\{$ flour, flour $\multimap$ bread $\},\{$ shop $\}) \in \mathrm{ch}_{b} \times \mathrm{ch}_{a}$ would satisfy them both. 


\begin{tabular}{|c|cc|}
\hline$a$ & $\emptyset$ & $\{$ elec $\}$ \\
\hline$\emptyset$ & $\emptyset \boldsymbol{~}$ & elec $\}$ \\
$\left\{\mathrm{H}_{2} \mathrm{O}\right\}$ & $\left\{\mathrm{H}_{2} \mathrm{O}\right\}$ & $\left\{\mathrm{H}_{2} \mathrm{O}\right.$, elec $\}$ \\
$\left\{\mathrm{H}_{2} \mathrm{O}, \mathrm{H}_{2} \mathrm{O}\right\}$ & $\left\{\mathrm{H}_{2} \mathrm{O}, \mathrm{H}_{2} \mathrm{O}\right\}$ & $\left\{\mathrm{H}_{2} \mathrm{O}, \mathrm{H}_{2} \mathrm{O}\right.$, elec $\}: \gamma_{f}$ \\
$\left\{\mathrm{H}_{2} \mathrm{O}, \mathrm{H}_{2} \mathrm{O}, \mathrm{H}_{2} \mathrm{O}\right\}$ & $\left\{\mathrm{H}_{2} \mathrm{O}, \mathrm{H}_{2} \mathrm{O}, \mathrm{H}_{2} \mathrm{O}\right\}$ & $\left\{\mathrm{H}_{2} \mathrm{O}, \mathrm{H}_{2} \mathrm{O}, \mathrm{H}_{2} \mathrm{O}\right.$, elec $\}: \gamma_{f}$ \\
$\{$ drink & $\{$ drink $\square$ & $\{$ drink, elec $\}$ \\
$\left\{\right.$ drink, $\left.\mathrm{H}_{2} \mathrm{O}\right\}$ & $\left\{\right.$ drink, $\left.\mathrm{H}_{2} \mathrm{O}\right\}$ & $\left\{\right.$ drink, $\mathrm{H}_{2} \mathrm{O}$, elec $\}$ \\
$\left\{\right.$ drink, $\left.\mathrm{H}_{2} \mathrm{O}, \mathrm{H}_{2} \mathrm{O}\right\}$ & $\left\{\right.$ drink, $\left.\mathrm{H}_{2} \mathrm{O}, \mathrm{H}_{2} \mathrm{O}\right\}$ & $\left\{\right.$ drink, $\mathrm{H}_{2} \mathrm{O}, \mathrm{H}_{2} \mathrm{O}$, elec $\}: \gamma_{f}$ \\
$\left\{\right.$ drink, $\left.\mathrm{H}_{2} \mathrm{O}, \mathrm{H}_{2} \mathrm{O}, \mathrm{H}_{2} \mathrm{O}\right\}$ & $\left\{\right.$ drink, $\left.\mathrm{H}_{2} \mathrm{O}, \mathrm{H}_{2} \mathrm{O}, \mathrm{H}_{2} \mathrm{O}\right\}$ & $\left\{\right.$ drink, $\mathrm{H}_{2} \mathrm{O}, \mathrm{H}_{2} \mathrm{O}, \mathrm{H}_{2} \mathrm{O}$, elec $\} \mathbf{\square}: \gamma_{a}, \gamma_{f}$ \\
\hline
\end{tabular}

Figure 8: The game $G_{a f}^{\epsilon^{\prime \prime}}$.

\begin{tabular}{|c|cc|}
\hline$b$ & $\emptyset$ & $\{$ shop $\}$ \\
\hline$\emptyset$ & $\emptyset$ & $\{$ shop $\}: \gamma_{b}$ \\
$\{$ flour $\}$ & $\{$ flour $\}$ & $\{$ flour, shop $\}: \gamma_{b}$ \\
$\{$ flour $\multimap$ bread $\}$ & $\{$ flour $\multimap$ bread $\}$ & $\{$ flour $\multimap$ bread, shop $\}: \gamma_{b}$ \\
$\{$ flour, flour $\}$ & $\{$ flour, flour $\}$ & $\{$ flour, flour, shop $\}: \gamma_{b}$ \\
$\{$ flour, flour $\multimap$ bread $\}$ & $\{$ flour, flour $\multimap$ bread $\}: \gamma_{a}$ & $\{$ flour, flour $\multimap$ bread, shop $\}: \gamma_{b}, \gamma_{a}$ \\
$\{$ flour, flour, flour $\multimap$ bread $\}$ & $\{$ flour, flour, flour $\multimap$ bread $\}: \gamma_{a}$ & $\{$ flour, flour, flour $\multimap$ bread, shop $\}: \gamma_{b}, \gamma_{a}$ \\
\hline
\end{tabular}

Figure 9: The game $G_{a b}^{\epsilon}$. Bernard plays rows, and Ann plays columns. The profile $(\emptyset, \emptyset) \in \mathrm{ch}_{b} \times \mathrm{ch}_{a}$ is the only Nash equilibrium in presence of parsimonious preferences.

A desirable redistribution. So the arbitrator redistributes the resources that are available. He assigns the bread making equipment and half the flour to Ann. He assigns the lease agreement and half the flour to Bernard. That is, $\epsilon_{a}^{\prime}=\{$ flour, flour $\multimap$ bread $\}$ and $\epsilon_{b}^{\prime}=\{$ flour, shop $\}$. This redistribution yields the game $G_{a b}^{\epsilon^{\prime}}$. It can be depicted as on Figure 10. In $G_{a b}^{\epsilon^{\prime}}$, the profile $(\emptyset, \emptyset)$ is not a Nash equilibrium, and so has been eliminated from $G_{a b}^{\epsilon}$. Indeed, it does not satisfy Bernard, and he has an incentive to deviate to the profile $(\{$ shop $\}, \emptyset) \in \mathrm{ch}_{b} \times \mathrm{ch}_{a}$ in which his objective is satisfied. But $(\{$ shop $\}, \emptyset)$ is not a Nash equilibrium either. Indeed, it does not satisfy Ann, and she has an incentive to deviate to the profile $(\{$ shop $\},\{$ flour, flour $\multimap$ bread $\}) \in \mathrm{ch}_{b} \times \mathrm{ch}_{a}$. From here, nobody has an incentive to deviate, and it is a Nash equilibrium. It is in fact the only Nash equilibrium in $G_{a b}^{\epsilon^{\prime}}$.

One can readily see that the profile ( $\{$ flour, shop $\},\{$ flour, flour $\multimap$ bread $\}) \in \mathrm{ch}_{b} \times \mathrm{ch}_{a}$, even though it satisfies both Ann and Bernard, is not a Nash equilibrium. Bernard has an incentive to provide less resources. The same can be said about the profile $(\{$ flour, shop $\},\{$ flour $\multimap$ bread $\}) \in \mathrm{ch}_{b} \times \mathrm{ch}_{a}$. 


\begin{tabular}{|c|c|c|c|c|}
\hline$b>a$ & $\emptyset$ & $\{$ flour $\}$ & $\{$ flour $\multimap$ bread $\}$ & $\{$ flour, flour $\multimap$ bread $\}$ \\
\hline$\emptyset$ & $\emptyset$ & $\{$ flour $\}$ & $\{$ flour $\multimap$ bread $\}$ & $\{$ flour, flour $\multimap$ bread $\}: \gamma_{a}$ \\
\hline$\{$ flour $\}$ & $\{$ flour $\}$ & $\{$ flour, flour $\}$ & $\{$ flour, flour $\multimap$ bread $\}: \gamma_{a}$ & $\{$ flour, flour, flour $\multimap$ bread $\}: \gamma_{a}$ \\
\hline$\{$ shop $\}$ & $\{$ shop $\}: \gamma_{b}$ & $\{$ flour, shop $\}: \gamma_{b}$ & $\{$ flour $\multimap$ bread, shop $\}: \gamma_{b}$ & $\{$ flour, flour $\multimap$ bread, shop $\}: \gamma_{b}, \gamma_{a}$ \\
\hline$\{$ flour, shop $\}$ & $\{$ flour, shop $\}: \gamma_{b}$ & $\{$ flour, flour, shop $\}: \gamma_{b}$ & $\{$ flour, flour $\multimap$ bread, shop $\}: \gamma_{b}, \gamma_{a}$ & $\{$ flour, flour, flour $\multimap$ bread, shop $\}: \gamma_{b}, \gamma_{a}$ \\
\hline
\end{tabular}

Figure 10: The game $G_{a b}^{\epsilon^{\prime}}$. The profile $(\{$ shop $\},\{$ flour, flour $\multimap$ bread $\}) \in \mathrm{ch}_{b} \times \mathrm{ch}_{a}$ is the only Nash equilibrium in presence of parsimonious preferences.

\subsection{An interconnected economy}

We present a three-player variant of Example 1 The setting, which we remind briefly, is analogous. In a local telecom industry, three companies must by regulation accept traffic from each other's customers. Moreover, Activating a network at some capacity has a cost, and companies can privately activate and deactivate networks on the fly.

Company $A$ manages a $3 \mathrm{G}$ network of comprised capacity 3 (bundled as capacities 1 , and 2). Company $B$ manages a $4 \mathrm{G}$ network of capacity 3 (bundled as capacities 1 , and 2). Company $A$ need to offer their customers $3 \mathrm{G}$ at capacity 2 and $4 \mathrm{G}$ at capacity 1 . Company $B$ need to offer their customers $3 \mathrm{G}$ at capacity 2 and $4 \mathrm{G}$ at capacity 2 .

A new company, Company $C$ is entering in this interconnected economy. It has some capital, say, two token of an arbitrary unit; one token being fair price for a mobile network antenna. However, Company $C$ does not manage any network. Company $C$ needs to offer their customers $3 \mathrm{G}$ at capacity 1 and $4 \mathrm{G}$ at capacity 1.

Again, we will only consider parsimonious preferences and assume that LOG is MULT. Since we are using this modest fragment, we trust that formal proofs would be more than superfluous and will be omitted.

We introduce the resources involved in the scenario:

- the resource of one capacity of $3 \mathrm{G}$ network: $3 \mathrm{G}$

- the resource of one capacity of $4 \mathrm{G}$ network: $4 \mathrm{G}$

- the resource of one token of capital: cap

Game definition. Let $G_{i e}^{\epsilon}=\left(\{a, b, c\}, \gamma_{a}, \gamma_{b}, \gamma_{c}, \epsilon_{a}, \epsilon_{b}, \epsilon_{c}\right)$ be the individual resource game with three players, Company $A, B$, and $C$ being represented by $a, b$, and $c$, respectively .

In the game $G_{i e}^{\epsilon}$, we have $\epsilon_{a}=\{3 \mathrm{G}, 3 \mathrm{G} \otimes 3 \mathrm{G}\} . \epsilon_{b}=\{4 \mathrm{G}, 4 \mathrm{G} \otimes 4 \mathrm{G}\}$, and $\epsilon_{c}=\{$ cap, cap $\}$ for endowments. The objectives are as follows: $\gamma_{a}=3 \mathrm{G} \otimes 3 \mathrm{G} \otimes 4 \mathrm{G}, \gamma_{b}=3 \mathrm{G} \otimes 3 \mathrm{G} \otimes 4 \mathrm{G} \otimes 4 \mathrm{G}$, and $\gamma_{c}=3 \mathrm{G} \otimes 4 \mathrm{G}$.

Two equilibria. The game $G_{i e}^{\epsilon}$ and the realized objectives can be depicted as on Figure 11 Company $B$, Player $b$, plays rows, Company $A$, Player $a$, plays column. For simplicity we do not represent all Company $C$ 's choices because they do not bear on the players' objectives. We only represent Player $c$ 's choice $\emptyset$. With other choices different from $\emptyset$, the realized objectives are exactly the same. Assuming parsimonious preferences, no profile where Company $C$ 's action is different from $\emptyset$ is a Nash equilibrium.

There are two Nash equilibria in the IRG $G_{i e}^{\epsilon}$, namely, $(\emptyset, \emptyset, \emptyset)$ and $(\{3 \mathrm{G} \otimes 3 \mathrm{G}\},\{4 \mathrm{G} \otimes 4 \mathrm{G}\}, \emptyset)$. In the latter, all agents realize their objective. In the former, none of them do.

Eliminating the bad equilibrium. In the IRG $G_{i e}^{\epsilon}$, the profile $(\emptyset, \emptyset, \emptyset)$ is an arguably undesirable equilibrium. An arbitrator could however advise the three companies to redistribute their endowments to 


\begin{tabular}{|c|cccc|}
\hline$c$ & \multicolumn{4}{|c|}{$\emptyset$} \\
\hline$b$ & $\emptyset$ & $\{3 G\}$ & $\{3 \mathrm{G} \otimes 3 \mathrm{G}\}$ & $\{3 \mathrm{G}, 3 \mathrm{G} \otimes 3 \mathrm{G}\}$ \\
\hline$\emptyset$ & $\emptyset \boldsymbol{⿴ 囗 十}$ & $\{3 \mathrm{G}\}$ & $\{3 \mathrm{G} \otimes 3 \mathrm{G}\}$ & $\{3 \mathrm{G}, 3 \mathrm{G} \otimes 3 \mathrm{G}\}$ \\
$\{4 \mathrm{G}\}$ & $\{4 \mathrm{G}\}$ & $\{4 \mathrm{G}, 3 \mathrm{G}\}: \gamma_{c}$ & $\{4 \mathrm{G}, 3 \mathrm{G} \otimes 3 \mathrm{G}\}: \gamma_{a}, \gamma_{c}$ & $\{4 \mathrm{G}, 3 \mathrm{G}, 3 \mathrm{G} \otimes 3 \mathrm{G}\}: \gamma_{a}, \gamma_{c}$ \\
$\{4 \mathrm{G} \otimes 4 \mathrm{G}\}$ & $\{4 \mathrm{G} \otimes 4 \mathrm{G}\}$ & $\{4 \mathrm{G} \otimes 4 \mathrm{G}, 3 \mathrm{G}\}: \gamma_{c}$ & $\{4 \mathrm{G} \otimes 4 \mathrm{G}, 3 \mathrm{G} \otimes 3 \mathrm{G}\}: \gamma_{a}, \gamma_{b}, \gamma_{c} \square$ & $\{4 \mathrm{G} \otimes 4 \mathrm{G}, 3 \mathrm{G}, 3 \mathrm{G} \otimes 3 \mathrm{G}\}: \gamma_{a}, \gamma_{b}, \gamma_{c}$ \\
$\{4 \mathrm{G}, 4 \mathrm{G} \otimes 4 \mathrm{G}\}$ & $\{4 \mathrm{G}, 4 \mathrm{G} \otimes 4 \mathrm{G}\}$ & $\{4 \mathrm{G}, 4 \mathrm{G} \otimes 4 \mathrm{G}, 3 \mathrm{G}\}: \gamma_{c}$ & $\{4 \mathrm{G}, 4 \mathrm{G} \otimes 4 \mathrm{G}, 3 \mathrm{G} \otimes 3 \mathrm{G}\}: \gamma_{a}, \gamma_{b}, \gamma_{c}$ & $\{4 \mathrm{G}, 4 \mathrm{G} \otimes 4 \mathrm{G}, 3 \mathrm{G}, 3 \mathrm{G} \otimes 3 \mathrm{G}\}: \gamma_{a}, \gamma_{b}, \gamma_{c}$ \\
\hline
\end{tabular}

Figure 11: Partial representation of the game $G_{i e}^{\epsilon}$.

eliminate $(\emptyset, \emptyset, \emptyset)$. The arbitrator could propose the redistribution $\epsilon^{\prime}$ of $\epsilon$, where $\epsilon_{a}^{\prime}=\{3 \mathrm{G} \otimes 3 \mathrm{G}$, cap $\}$, $\epsilon_{b}^{\prime}=\{4 \mathrm{G} \otimes 4 \mathrm{G}$, cap $\}$, and $\epsilon_{c}^{\prime}=\{3 \mathrm{G}, 4 \mathrm{G}\}$.

The game $G_{i e}^{\epsilon^{\prime}}$ and the realized objectives can be depicted as on Figure 12, when Player $c$ 's choice is $\emptyset$. The choices containing the resource cap are not represented. The resource cap has no bearing on the player's objectives, and no profile containing it is a Nash equilibrium.

\begin{tabular}{|c|ccc|}
\hline$c$ & \multicolumn{4}{|c|}{$\emptyset$} \\
\hline$b$ & $\emptyset$ & $\{3 \mathrm{G} \otimes 3 \mathrm{G}\}$ & $\cdots$ \\
\hline$\emptyset$ & $\emptyset$ & $\{3 \mathrm{G} \otimes 3 \mathrm{G}\}$ & $\ldots$ \\
$\{4 \mathrm{G} \otimes 4 \mathrm{G}\}$ & $\{4 \mathrm{G} \otimes 4 \mathrm{G}\}$ & $\{4 \mathrm{G} \otimes 4 \mathrm{G}, 3 \mathrm{G} \otimes 3 \mathrm{G}\}: \gamma_{a}, \gamma_{b}, \gamma_{c} \boldsymbol{口}$ & $\ldots$ \\
$\vdots$ & $\ldots$ & $\ldots$ & $\ddots$ \\
\hline
\end{tabular}

Figure 12: Partial representation of the game $G_{i e}^{\epsilon^{\prime}}$.

After the redistribution, Company $C$ manages a $3 \mathrm{G}$ and a $4 \mathrm{G}$ network, both at capacity 1 . Activating both of them would be enough to satisfy Company $C$ 's objective. In $G_{i e}^{\epsilon^{\prime}}$, Player $c$ thus has an incentive to deviate from $(\emptyset, \emptyset, \emptyset)$. Hence, the arbitrator's advice permits the elimination of the bad equilibrium: $(\emptyset, \emptyset, \emptyset)$ is not a Nash equilibrium in $G_{i e}^{\epsilon^{\prime}}$.

In the profile $(\emptyset, \emptyset,\{3 G, 4 G\})$, Player 1 has an incentive to deviate and play $\{3 G \otimes 3 G\}$, in order to realize its objective.

In turn, in the profile $(\{3 G \otimes 3 G\}, \emptyset,\{3 G, 4 G\})$, Player 2 has an incentive to deviate and play $\{4 G \otimes$ $4 \mathrm{G}$ to realize its objective. (Player 3, by parsimony, has also an incentive to withdraw the resource $3 \mathrm{G}$.)

In the profile $(\{3 G \otimes 3 G\},\{4 G \otimes 4 G\},\{3 G, 4 G\})$, by parsimony, Player 3 has an incentive to deviate to the choice $\emptyset$.

Every player is satisfied in $(\{3 \mathrm{G} \otimes 3 \mathrm{G}\},\{4 \mathrm{G} \otimes 4 \mathrm{G}\}, \emptyset)$, and none of them have an incentive to withdraw any resources. Hence, the good equilibrium of $G_{i e}^{\epsilon},(\{3 \mathrm{G} \otimes 3 \mathrm{G}\},\{4 \mathrm{G} \otimes 4 \mathrm{G}\}, \emptyset)$, is still a Nash equilibrium in $G_{i e}^{\epsilon^{\prime}}$. In addition, this is the unique Nash equilibrium in $G_{i e}^{\epsilon^{\prime}}$.

\section{Conclusions}

We presented a class of games of resources that exploits the formalisms and reasoning methods for resource-sensitive logics. The language of Linear Logic allows us to represent in an harmonious way simultaneous resources, deterministic and non-deterministic choice, and resource-transforming capacities. 


\begin{tabular}{|c|c|c|c|}
\hline & & linear & affine \\
\hline \multirow{3}{*}{ 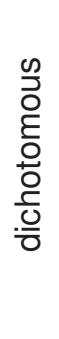 } & $\mathrm{NE}$ & $\begin{array}{l}\text { NP-hard (Prop. 11) } \\
\text { in } \Pi_{2}^{p} \text { (Prop. 12) }\end{array}$ & $\begin{array}{l}\text { NP-hard (Prop. 11) } \\
\text { in } P^{N P \| I} \text { (Prop. 15) }\end{array}$ \\
\hline & $\mathrm{RE}$ & $\begin{array}{l}\text { coNP-hard (Prop. 20) } \\
\text { in } \Sigma_{2}^{\mathrm{p}} \text { (Prop. 18) }\end{array}$ & $\begin{array}{l}\text { coNP-hard (Prop.20 } \\
\text { in PNP\|I (Prop.19) }\end{array}$ \\
\hline & $\mathrm{RC}$ & $\begin{array}{l}\text { NP-hard (Prop. 21) } \\
\text { in } \Sigma_{3}^{\mathrm{p}} \text { (Prop. 221) }\end{array}$ & $\begin{array}{l}\text { NP-hard (Prop. 21) } \\
\text { in } \Sigma_{2}^{\mathrm{p}} \text { (Prop. 23) }\end{array}$ \\
\hline \multirow{3}{*}{ 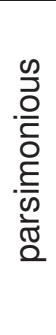 } & NE & $\begin{array}{l}\text { coNP-hard (Prop. 28) } \\
\text { in } \Pi_{2}^{\text {p }} \text { (Prop. 29) }\end{array}$ & $\begin{array}{l}\text { coNP-hard (Prop.28 } \\
\text { in PNP\| (Prop. 31) }\end{array}$ \\
\hline & RE & $\begin{array}{l}\text { coNP-hard (Prop. 34) } \\
\text { in } \Sigma_{2}^{\mathrm{p}} \text { (Prop. 32) }\end{array}$ & $\begin{array}{l}\text { coNP-hard (Prop. } 34 \\
\text { in } P^{N P \|} \text { (Prop 33) }\end{array}$ \\
\hline & $\mathrm{RC}$ & $\begin{array}{l}\text { coNP-hard (Prop. 35) } \\
\text { in } \sum_{3}^{\mathrm{p}} \text { (Prop. 36) }\end{array}$ & $\begin{array}{l}\text { coNP-hard (Prop. 35) } \\
\text { in } \sum_{2}^{\mathrm{p}} \text { (Prop. 37) }\end{array}$ \\
\hline
\end{tabular}

Table 4: Complexity results when the problem of provability in LOG is in NP.

In individual resource games, each player of a game is endowed with a multiset of resources and has an objective represented by a resource. In this context, we studied three decision problems, the first of which is to decide whether a profile is a Nash equilibrium. Some profiles that are not equilibria can have desirable outcomes from the point of view of an external authority. Some equilibria can have outcomes that are undesirable. We thus studied redistribution schemes which can be used by a central authority to enforce some behavior, either by disincentivizing a behavior or incentivizing a behavior. This yielded two related decision problems: rational elimination and rational construction of profiles. We illustrated the models and the decision problems with two examples.

We considered dichotomous or parsimonious preferences, and showed striking algorithmic differences when the logic employed admits or not the weakening rule.

Summary of the complexity results. For all decision problems, for both types of preferences, we have studied six cases where LOG can have the following properties along two dimensions: (1) affine vs. linear, and (2) in PTIME vs. in NP vs. in PSPACE.

When LOG is NP-complete, we sum up precisely the results in Table 4. For instance, one can quickly gather that when LOG is Affine MLL (whose sequent provability is NP-complete) and we consider parsimonious preferences, RATIONAL ELIMINATION is in $\mathrm{P}^{\mathrm{NP} \|}$. We proved the same problem to be in $\Sigma_{2}^{\mathrm{p}}$ when LOG is Linear MLL. When LOG is in PTIME, we sum up precisely the results in Table 5 , We thus obtained some positive results when the resources are expressed in the fragment MULT, which is suitable to represent and reason about multisets of resources.

Theorem 38. When LOG is Affine MULT, with dichotomous or parsimonious preferences, the problems NASH EQUILIBRIUM and RATIONAL ELIMINATION can be solved in polynomial time. 


\begin{tabular}{|c|c|c|c|}
\hline & & linear & affine \\
\hline \multirow{3}{*}{ 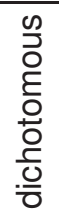 } & $\mathrm{NE}$ & in coNP (Prop.12) & in PTIME (Prop. 15) \\
\hline & $\mathrm{RE}$ & in NP (Prop. 18) & in PTIME (Prop.19) \\
\hline & $\mathrm{RC}$ & in $\Sigma_{2}^{\mathrm{p}}$ (Prop. 22) & in NP (Prop. 23) \\
\hline \multirow{3}{*}{ 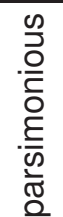 } & $\mathrm{NE}$ & in coNP (Prop. 29) & in PTIME (Prop. 31) \\
\hline & $\mathrm{RE}$ & in NP (Prop. 32) & in PTIME (Prop 33) \\
\hline & $\mathrm{RC}$ & in $\Sigma_{2}^{\mathrm{p}}$ (Prop. 36) & in NP (Prop. 37) \\
\hline
\end{tabular}

Table 5: Complexity results when the problem of provability in LOG is in PTIME.

It is interesting to note that, although weakening usually does not change the complexity of the problem of sequent provability of the logics we considered 7 we have always been able to capitalize on its presence to simplify our solutions to the problems we studied here.

Putting the results of this paper together, it is also easy to see that we have this theorem.

Theorem 39. When LOG is MALL, linear or affine, with dichotomous or with parsimonious preferences, all three decision problems are PSPACE-complete.

First-Order MLL is one of these logics whose complexity of sequent provability is in NP. On the other hand, sequent provability for First-Order MALL is NEXPTIME-complete. It is routine to adapt our proofs to show this theorem.

Theorem 40. When LOG is First-Order MALL, linear or affine, with dichotomous or with parsimonious preferences, all three decision problems are NEXPTIME-complete.

Comparison with the related literature. The research in artificial intelligence, multiagent systems, and computer science has shown some interest in the formal and computational aspects of resourceconscious agents (e.g., [17, 47, 33, 10, 18, 44, 35, 1, 43]).

Boolean games [19, 4] are games based on classical logic. Each player controls a set of Boolean variables and produces truth values which can be used without restriction towards the Boolean goals, expressed as classical propositional formula. Somehow, also in Boolean games do the players produce and consume 'resources'. But there are no immediate natural correspondences between IRGs and Boolean games. As in Boolean games, we could force the endowments to be non-overlapping (for exclusive control over a resource). Moreover, we could allow the players in our games to have preferences about the absence of a resource. Under these conditions, and using classical propositional logic as LOG, a connection would then exist.

Electric Boolean games [18] are an extension of Boolean games where playing a certain action has a numeric cost, and agents are endowed with a certain amount of 'energy'. Deciding whether a profile is a Nash equilibrium in a Boolean game is coNP-complete [4]. In electric Boolean games, deciding

\footnotetext{
${ }^{7}$ We did not consider full propositional Linear Logic, which also contains so-called 'exponentials'. Weakening does make a difference: sequent provability in full propositional Linear Logic is undecidable [26], while sequent provability in full propositional Affine Logic is decidable [22].
} 
whether a profile is rationally eliminable is NP-complete, while deciding whether a profile is rationally constructible is coNP-hard and in $\Delta_{2}^{\mathrm{p}}$.

In Boolean games, goals of players are expressed as classical propositional formulas. Moreover, game outcomes or profiles are in fact models of classical propositional logic, i.e., valuations. Checking whether the goal of a player is satisfied in a game profile is thus an easy problem in Boolean games. This is also true in electric Boolean games. In contrast in resource games, checking whether the goal of a player is satisfied in a game profile is as hard as provability in LOG.

Unsurprisingly, when working with the fragments MLL or MALL, the trend is that the complexity of decision problems in individual resource games is higher than for their counterparts in electric Boolean games. An obvious exception is the problem to decide whether an individual resource game admits a Nash equilibrium when LOG is affine and we consider dichotomous preferences. The problem is trivial by Proposition 14 (there is always a Nash equilibrium), while it is $\Sigma_{2}^{\mathrm{p}}$-complete in Boolean games [4].

Moreover, in individual resource games, there is no one-to-one correspondence between profiles and outcomes. This is another difference with electric Boolean game. As a consequence, the notions of elimination and construction in individual resource games add a bit of complexity by having to consider a set of profiles with the same outcomes.

On the other hand, the fragment MULT is one instance of LOG in which it is easy to check whether a goal of a player is satisfied in a game profile (Proposition 7). In this context, and as shown on Table 5 and compared to the realm of Boolean games, reasoning about IRGs remains a relatively easy task. It can even be tractable if one considers Affine MULT. Affine logic should be used when we can assume that a player satisfied with an outcome would be satisfied with a more sizeable outcome, which is often a very acceptable assumption.

Congestion games (CGs) [39] (see also Potential Games [27]; exact potential games correspond to CGs up to an isomorphism) are a celebrated class of games where the players interact in resource-sensitive environments. Despite some apparent similarities between IRGs and CGs, they are rather superficial. Players in CGs do not have endowments per se. Players' actions in CGs consist in choosing a subset of an already available common pool of resources to use. In CGs, the players are only consumers. In IRGs, players are consumers but also producers of resources; their actions consist in making resources available in the common pool. In CGs, these resources are exclusively atomic resources while in IRGs they can be any logical formula in LOG.

With the decision problems of rational elimination and rational construction, there is a dimension of social choice theory and mechanism design. Formal frameworks concerned with redistribution schemes and economic policies can be found for instance in [18] again, or [11, 25, 28].

Our games bear some resemblance with combinatorial exchanges [23] and with mixed multi-unit combinatorial auctions (MMUCAs) [8, 15], where the agents can be both sellers and buyers. Interestingly, in MMUCAs, sets of goods can be transformed into different sets of goods. Resource-transforming capacities are central, as the agents are allowed to bid on transformation services. Determining the sequences of bids to be accepted by an auctioneer is generally intractable in MMUCAs; [13] identifies tractable classes for the winner determination problem.

Finally, we focused on individual games and looked at Nash equilibria. Nonetheless, the setting allows one to easily build classes of coalition games, reminiscent of Coalitional Resource Games [47, 10] and of Coalition Skill Games [3]. In [43], we have started the study of what we called Rich Coalitional Resource Games (RCRGs). Individual Resource Games are essentially one-goal RCRGs.

Perspectives. We have obtained tight complexity results when LOG is PSPACE-complete. However, this is lacking when LOG is in NP and in PTIME. We suspect that the complexity of the diverse decision problems generally lie above the lower bounds we have obtained. It is more likely that some proposed upper bounds are tight. One perspective will thus be to investigate whether some decision problems could 
be proven hard for some complexity class in the polynomial or Boolean hierarchy, for instance using the techniques from [45] of raising NP lower bounds to lower bounds for classes above NP.

Resource games based on resource-sensitive logics become all the more significant when the resources are subject to transforming activities. We can exploit the existing research on these resource-sensitive logics about their proof theory. In particular, through the Curry-Howard correspondence between proofs and programs (see, e.g., [14]), an exciting perspective is the possibility to interpret the logical proofs as rigorous programs to be executed by the players. We can expect to obtain some results for the automated generation of plans, where the resources can be subjected to a series of transforming activities by the agents. Similar ideas have already been defended in multiagent systems (see, e.g., [24]).

Our models are agnostic about how the contributed resources are distributed. Instead of having preferences about a raw profile $P$, the player's preferences could be raised over the result of the (fair, envy-free, efficient, etc) allocation of the resources [5] contributed in out $(P)$. These are interesting extensions that are just a step away to get the models more fit for application, although at the expense of mathematical simplicity.

We are interested in using resource games in problems of gamification. Gamification refers to the broad application of game-design techniques in contexts that do not otherwise present game-like features [9, 36]. Gamification aims at incentivizing an intended behavior by introducing rewards for specific tasks. Rewards often present themselves as virtual resources such as achievement badges. Formally, they might be nothing more than distinguished tokens of resources. In Example 1, we saw that the profile where all companies refrain from providing any resources, $(\emptyset, \emptyset)$, is a Nash equilibrium. This is an undesirable behaviour that policy makers might be able to anticipate by using the analytical tools defined in this paper, and to avoid by using advanced gamification methods.

\section{Acknowledgments}

I thank an anonymous reviewer for making a number of suggestions that greatly helped to improve the presentation. I am grateful to Jamie Gabbay for his enthusiasm and his encouragements. 


\section{A Sequent rules of Affine MALL}

We present the sequent rules for Affine MALL. In what follows, $A, B, A_{0}$, and $A_{1}$ are formulas. $\Gamma, \Gamma^{\prime}$, $\Delta$, and $\Delta^{\prime}$ are sequences of zero or more formulas. A sequent rule has an upper and a lower part. The upper part is composed of zero, one, or two sequents. The lower part is composed of one sequent. If there is a proof of all the sequents of the upper part, then the rule can be used to obtain a proof of the sequent of the lower part.

Identities

$$
\frac{}{A \vdash A} \text { ax } \quad \frac{\Gamma, A \vdash \Delta \quad \Gamma^{\prime} \vdash A, \Delta^{\prime}}{\Gamma, \Gamma^{\prime} \vdash \Delta, \Delta^{\prime}} \mathrm{cut}
$$

Structural Rules

$$
\begin{array}{cc}
\frac{\Gamma, A, B, \Gamma^{\prime} \vdash \Delta}{\Gamma, B, A, \Gamma^{\prime} \vdash \Delta} \mathrm{E} & \frac{\Gamma \vdash \Delta, A, B, \Delta^{\prime}}{\Gamma \vdash \Delta, B, A, \Delta^{\prime}} \mathrm{E} \\
\frac{\Gamma \vdash \Delta}{\Gamma, A \vdash \Delta} \mathrm{W} & \frac{\Gamma \vdash \Delta}{\Gamma \vdash \Delta, A} \mathrm{~W}
\end{array}
$$

Negation

$$
\frac{\Gamma \vdash A, \Delta}{\Gamma, \sim A \vdash \Delta} L \sim \frac{\Gamma, A \vdash \Delta}{\Gamma \vdash \sim A, \Delta} R \sim
$$

Multiplicatives

$$
\begin{aligned}
& \otimes \mathrm{R} \frac{\Gamma \vdash A, \Delta \quad \Gamma^{\prime} \vdash B, \Delta^{\prime}}{\Gamma, \Gamma^{\prime} \vdash A \otimes B, \Delta, \Delta^{\prime}} \quad \frac{\Gamma, A, B \vdash \Delta}{\Gamma, A \otimes B \vdash \Delta} \otimes \mathrm{L} \\
& \ngtr \mathrm{L} \frac{\Gamma, A \vdash \Delta \quad \Gamma^{\prime}, B \vdash \Delta^{\prime}}{\Gamma, \Gamma^{\prime}, A \ngtr B \vdash \Delta, \Delta^{\prime}} \quad \frac{\Gamma \vdash A, B, \Delta}{\Gamma \vdash A \ngtr 8, \Delta} \ngtr \mathrm{R} \\
& \multimap \mathrm{L} \frac{\Gamma \vdash A, \Delta \quad \Gamma^{\prime}, B \vdash \Delta^{\prime}}{\Gamma, \Gamma^{\prime}, A \multimap B, \Delta \vdash \Delta^{\prime}} \quad \frac{\Gamma, A \vdash B, \Delta}{\Gamma \vdash A \multimap B, \Delta} \multimap \mathbf{R} \\
& \frac{\Gamma \vdash \Delta}{\Gamma, \mathbf{1} \vdash \Delta} 1 \mathrm{~L} \quad \frac{}{\vdash \mathbf{1}} 1 \mathrm{R} \quad \frac{\Gamma \vdash \Delta}{\perp \vdash} \perp \mathrm{L} \quad \frac{\Gamma \vdash \Delta, \perp}{\Gamma \vdash \Delta}
\end{aligned}
$$

Additives (In $\oplus \mathbf{R}$, and \& $\mathbf{L}, i$ stands for either 0 or 1.)

$$
\begin{array}{ccc}
\& \mathrm{R} \frac{\Gamma \vdash A, \Delta \quad \Gamma \vdash B, \Delta}{\Gamma \vdash A \& B, \Delta} & \frac{\Gamma, A_{i} \vdash \Delta}{\Gamma, A_{0} \& A_{1} \vdash \Delta} \& \mathrm{~L} \\
\oplus \mathrm{L} \frac{\Gamma, A \vdash \Delta \quad \Gamma, B \vdash \Delta}{\Gamma, A \oplus B \vdash \Delta} & \frac{\Gamma \vdash A_{i}, \Delta}{\Gamma \vdash A_{0} \oplus A_{1}, \Delta} \oplus \mathrm{R} \\
\frac{}{\Gamma \vdash \top, \Delta} \top \mathrm{R} & \frac{}{\Gamma, \mathbf{0} \vdash \Delta} \mathrm{OL}
\end{array}
$$




\section{B Elements of computational complexity}

We need to assume some familiarity with computational complexity. This appendix only introduces some elements of terminology and some definitions about complexity theory. The reader familiar with these notions can use this section for quick reference. Another reader can use it as a starting point and move to a more complete introduction. A classic introduction to computational complexity is [31]. All elementary complexity classes used in this paper are presented in [40].

A decision problem (or problem for short) is a problem that is posed as 'yes'/'no' question of the values of the input.

The class PTIME, also noted $\mathrm{P}$, is the class of decision problems that can be solved in deterministic polynomial time (wrt. the size of the input). The class NP is the class of problems that can be solved in non-deterministic polynomial time. The class PSPACE is the class of problems that can be solved using a polynomial amount of space. The complement of a decision problem is the decision problem resulting from reversing the 'yes' and 'no' answers. For every class of complexity C, we denote coC the class populated with the complements of the problems in $\mathrm{C}$. Given two classes of complexity $\mathrm{C}_{1}$ and $\mathrm{C}_{2}$, the class $C_{1}^{C_{2}}$ is the class of problems that are in $C_{1}$ if we assume the availability of an oracle to solve the problems in $C_{2}$. An oracle for $C_{2}$ is a black box capable to solve every problem in $C_{2}$ in a single operation. Queries to an oracle can be adaptive (also called serial), or non-adaptive (also called parallel). A query is adaptive when it depends on the answer of a previous query. Non-adaptive queries on the other hand, can be chosen in advance and computed from the start and are asked in parallel.

For every class of complexity $\mathrm{C}$, we denote $\mathrm{P}^{\mathrm{C}}$ (resp. NPC) the class of problems solvable on a deterministic (resp. non-deterministic) polynomial-time bounded oracle Turing machine using an oracle set $C$. We denote $\mathrm{P}^{\mathrm{C}}[\mathrm{k}]$ and $\mathrm{NP}^{\mathrm{C}}[\mathrm{k}]$ when at most $k$ adaptive queries to $\mathrm{C}$ can be used. We denote $\mathrm{P}^{\mathrm{C}||[\mathrm{k}]}$ and $\mathrm{NP}^{\mathrm{C} \|[\mathrm{k}]}$ when at most $k$ non-adaptive queries to $\mathrm{C}$ can be used.

We denote $\mathrm{P}^{\mathrm{C} \|}$ (resp. $\mathrm{NP}^{\mathrm{C} \|}$ ) the class of problems solvable on a deterministic (resp. non-deterministic) polynomial-time bounded oracle Turing machine with non-adaptive queries to $C$. The class $\mathrm{P}^{\mathrm{NP} \|}$ is also referred to as $\Theta_{2}^{p}$.

The polynomial hierarchy. The polynomial hierarchy contains a family of complexity classes that are smaller than PSPACE. The class $\mathrm{P}$ lies at the bottom of the polynomial hierarchy. Then, for every positive integer $i$, we can define $\Delta_{\mathrm{i}}^{\mathrm{p}}, \Sigma_{\mathrm{i}}^{\mathrm{p}}$, and $\Pi_{\mathrm{i}}^{\mathrm{p}}$ recursively as follows:

- $\Delta_{0}^{\mathrm{p}}=\Sigma_{0}^{\mathrm{p}}=\Pi_{0}^{\mathrm{p}}=\mathrm{P}$;

- $\Delta_{i+1}^{\mathrm{p}}=\mathrm{P}^{\Sigma_{\mathrm{i}}^{\mathrm{p}}}$;

- $\sum_{\mathrm{i}+1}^{\mathrm{p}}=N \mathrm{P}^{\Sigma_{\mathrm{i}}^{\mathrm{p}}}$;

- $\Pi_{\mathrm{i}}^{\mathrm{p}}=\operatorname{co} \sum_{\mathrm{i}}^{\mathrm{p}}$.

The Boolean hierarchy over NP. The Boolean hierarchy has been studied in [46, 21, 45]. The Boolean hierarchy over NP contains a family of complexity classes that are smaller than $\Delta_{2}^{p}$. The class NP lies at the bottom of the Boolean hierarchy over NP. Here, we are better off looking at complexity classes not as classes of decision problems, but as classes of languages. A language is the formal realization of a decision problem. Let $p$ be a decision problem with $k$ inputs. A language of $p$ is the language $L_{p}=$ $\left\{\left(a_{1}, \ldots, a_{k}\right) \mid p\right.$ answers 'yes' of the input $\left.\left(a_{1}, \ldots, a_{k}\right)\right\}$. Given a class of complexity $\mathrm{C}$, we say that $L_{p} \in \mathrm{C}$ iff $p \in \mathrm{C}$. Then, given two classes of complexity $\mathrm{C}_{1}$ and $\mathrm{C}_{2}$, each representing a set of languages and the decision problems they formalize, we define $C_{1} \wedge C_{2}=\left\{L_{1} \cap L_{2} \mid L_{1} \in C_{1}\right.$ and $\left.L_{2} \in C_{2}\right\}$ and $\mathrm{C}_{1} \vee \mathrm{C}_{2}=\left\{L_{1} \cup L_{2} \mid L_{1} \in \mathrm{C}_{1}\right.$ and $\left.L_{2} \in \mathrm{C}_{2}\right\}$. In this context, the class NP is the class of languages that can be recognised in non-deterministic polynomial time. Then, for every positive integer $i$, we can define $\mathrm{BH}_{\mathrm{i}}$ recursively as follows: 
- $\mathrm{BH}_{0}=\mathrm{NP}$

- $\mathrm{BH}_{2 \mathrm{k}}=\mathrm{coNP} \wedge \mathrm{BH}_{2 \mathrm{k}-1}$;

- $\mathrm{BH}_{2 \mathrm{k}+1}=\mathrm{NP} \vee \mathrm{BH}_{2 \mathrm{k}}$.

The class $\mathrm{BH}_{2}=\mathrm{NP} \wedge$ coNP is the "difference class" $\mathrm{D}^{\mathrm{P}}$ presented in [32].

Useful properties. Besides the definitions, the following properties are useful:

- $\mathrm{C}_{1}^{\mathrm{co} \mathrm{C}_{2}}=\mathrm{C}_{1}^{\mathrm{C}_{2}}$ (for all two classes $\mathrm{C}_{1}$ and $\mathrm{C}_{2}$ );

- $N P^{\Sigma_{i}^{p}}=\Sigma_{i+1}^{p}$;

- $\operatorname{co} \Sigma_{i}^{\mathrm{p}}=\Pi_{\mathrm{i}}^{\mathrm{p}}$

- $N \Delta_{i}^{p}=\Sigma_{i}^{p}$

- $P^{\Delta_{i}^{p}}=\Delta_{i}^{p}$

- $\Sigma_{i}^{\mathrm{P}} \subseteq$ PSPACE;

- PSPACE $=$ coPSPACE $=P^{\text {PSPACE }}=$ NPPSPACE;

- $\mathrm{BH}_{\mathrm{i}} \subseteq \Delta_{2}^{\mathrm{p}}$;

- $\mathrm{P}^{\mathrm{NP} \|[\mathrm{k}]} \subseteq \mathrm{BH}_{\mathrm{k}+1} \subseteq \mathrm{P}^{\mathrm{NP} \|[\mathrm{k}+1]}$.

\section{Proofs of the realized objectives in the Example of Section 6.1}

The proof of $\mathrm{H}_{2} \mathrm{O}, \mathrm{H}_{2} \mathrm{O}$, elec $\vdash \gamma_{f}$ will be instrumental for the subsequent proofs. We label it Proof $\star$ for reuse.

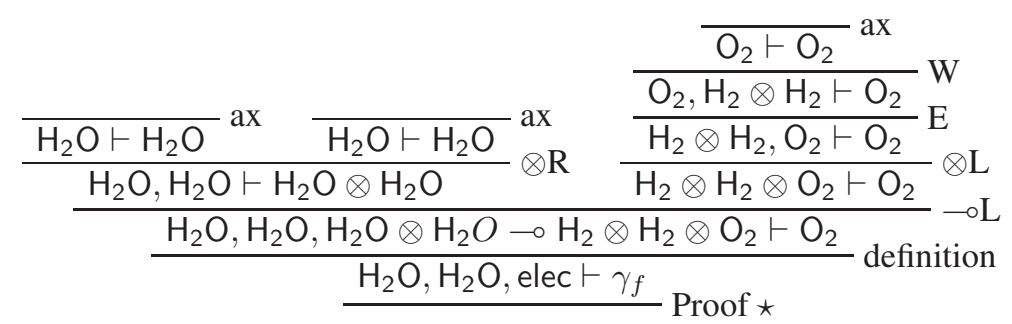

The other realized objectives of the fish are immediate using Proof $\star$ and the weakening rule. We prove that $\mathrm{H}_{2} \mathrm{O}, \mathrm{H}_{2} \mathrm{O}, \mathrm{H}_{2} \mathrm{O}$, elec $\vdash \gamma_{f}$, drink, $\mathrm{H}_{2} \mathrm{O}, \mathrm{H}_{2} \mathrm{O}$, elec $\vdash \gamma_{f}$, and drink, $\mathrm{H}_{2} \mathrm{O}, \mathrm{H}_{2} \mathrm{O}, \mathrm{H}_{2} \mathrm{O}$, elec $\vdash \gamma_{f}$.

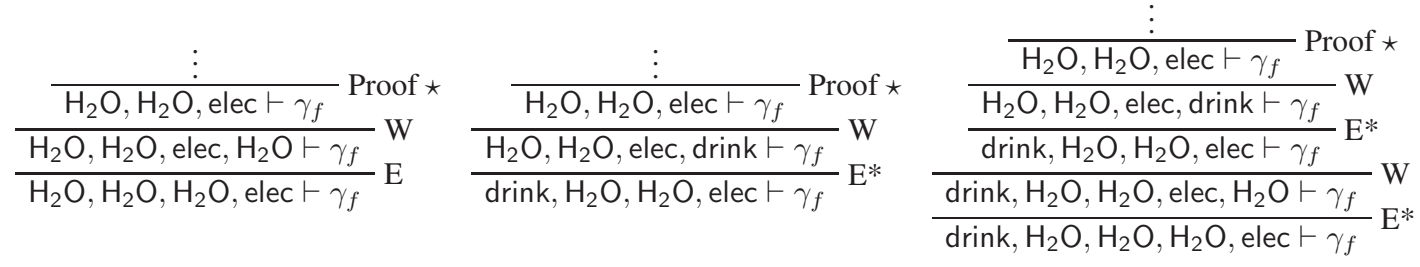

Finally, we prove drink, elec, $\mathrm{H}_{2} \mathrm{O}, \mathrm{H}_{2} \mathrm{O}, \mathrm{H}_{2} \mathrm{O} \vdash \gamma_{a}$. The proof also uses Proof $\star$.

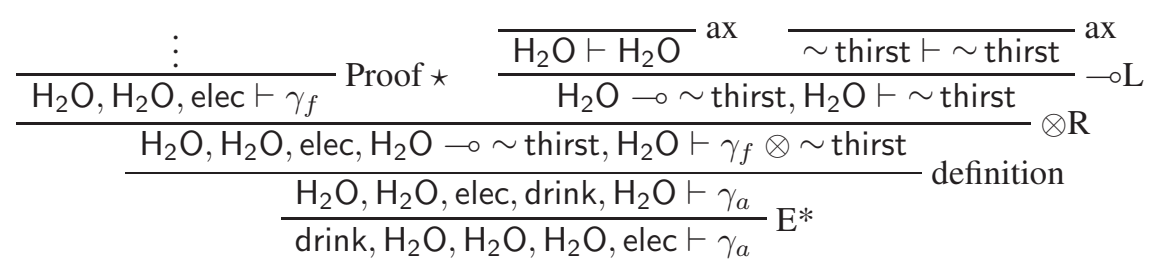




\section{References}

[1] Natasha Alechina, Nils Bulling, Brian Logan, and Hoang Nga Nguyen. The virtues of idleness: A decidable fragment of resource agent logic. Artificial Intelligence, 245:56-85, 2017.

[2] Jens Ambak, Bridger Mitchell, Werner Neu, Karl-Heinz Neumann, Ingo Vogelsang, Godefroy Dang N'Guyen, and Bernd Ickenroth. Network interconnection in the domain of ONP. Final report, November 1994, 1994.

[3] Yoram Bachrach, David C. Parkes, and Jeffrey S. Rosenschein. Computing Cooperative Solution Concepts in Coalitional Skill Games. Artificial Intelligence, 204:1-21, 2013.

[4] Elise Bonzon, Marie-Christine Lagasquie-Schiex, Jérôme Lang, and Bruno Zanuttini. Boolean Games Revisited. In 17th European Conference on Artificial Intelligence (ECAI'06), volume 141 of Frontiers in Artificial Intelligence and Applications, pages 265-269. IOS Press, 2006.

[5] Sylvain Bouveret, Yann Chevaleyre, and Nicolas Maudet. Fair Allocation of Indivisible Goods, chapter 12, pages 284-310. In Brandt et al. [6], 2016.

[6] Felix Brandt, Vincent Conitzer, Ulle Endriss, Jérôme Lang, and Ariel D. Procaccia, editors. Handbook of Computational Social Choice. Cambridge University Press, New York, NY, USA, 2016.

[7] W. Gerald Brock. The Economics of Interconnection. Technical report, Teleport Communication Group, 1995. Prepared for Teleport Communications Group.

[8] Jesús Cerquides, Ulle Endriss, Andrea Giovannucci, and Juan A. Rodríguez-Aguilar. Bidding languages and winner determination for mixed multi-unit combinatorial auctions. In Proceedings of the 20th International Joint Conference on Artifical Intelligence, IJCAI'07, pages 1221-1226, San Francisco, CA, USA, 2007. Morgan Kaufmann Publishers Inc.

[9] Sebastian Deterding, Miguel Sicart, Lennart Nacke, Kenton O'Hara, and Dan Dixon. Gamification. using game-design elements in non-gaming contexts. In CHI'11 Extended Abstracts on Human Factors in Computing Systems, CHI EA'11, pages 2425-2428, New York, NY, USA, 2011. ACM.

[10] Paul E. Dunne, Sarit Kraus, Efrat Manisterski, and Michael Wooldridge. Solving coalitional resource games. Artificial Intelligence, 174(1):20-50, 2010.

[11] Ulle Endriss, Sarit Kraus, Jérôme Lang, and Michael Wooldridge. Designing Incentives for Boolean Games. In 10th International Conference on Autonomous Agents and Multiagent Systems, AAMAS'11, pages 79-86. International Foundation for Autonomous Agents and Multiagent Systems, 2011.

[12] Shaheen Fatima, Sarit Kraus, and Michael J. Wooldridge. Principles of Automated Negotiation. Cambridge University Press, 2014.

[13] Valeria Fionda and Gianluigi Greco. The complexity of mixed multi-unit combinatorial auctions: Tractability under structural and qualitative restrictions. Artificial Intelligence, 196:1 - 25, 2013.

[14] Dov M. Gabbay and Ruy J. G. B. de Queiroz. Extending the Curry-Howard Interpretation to Linear, Relevant and Other Resource Logics. Journal of Symbolic Logic, 57(4):1319-1365, 1992.

[15] Andrea Giovannucci, Jesús Cerquides, Ulle Endriss, and Juan A. Rodríguez-Aguilar. A graphical formalism for mixed multi-unit combinatorial auctions. Autonomous Agents and Multi-Agent Systems, 20(3):342-368, May 2010. 
[16] Jean-Yves Girard. Linear logic. Theoretical Computer Science, 50(1):1-101, 1987.

[17] James Harland and Michael Winikoff. Agent negotiation as proof search in linear logic. In The First International Joint Conference on Autonomous Agents \& Multiagent Systems, AAMAS 2002, Proceedings, pages 938-939, 2002.

[18] Paul Harrenstein, Paolo Turrini, and Michael Wooldridge. Electric Boolean Games: Redistribution Schemes for Resource-Bounded Agents. In 14th International Conference on Autonomous Agents and Multi-agent Systems, AAMAS'15, pages 655-663, 2015.

[19] Paul Harrenstein, Wiebe van der Hoek, John-Jules Meyer, and Cees Witteveen. Boolean games. In Proceedings of the 8th Conference on Theoretical Aspects of Rationality and Knowledge, TARK'01, pages 287-298, San Francisco, CA, USA, 2001. Morgan Kaufmann Publishers Inc.

[20] Max I. Kanovich. The complexity of Horn fragments of Linear Logic. Annals of Pure and Applied Logic, 69(2-3):195-241, 1994.

[21] Johannes Köbler, Uwe Schöning, and Klaus W. Wagner. The difference and truth-table hierarchies for NP. Theoretical Informatics and Applications, 21:419-435, 1987.

[22] A. P. Kopylov. Decidability of Linear Affine Logic. Information and Computation, 164(1):173-198, 2001.

[23] Anshul Kothari, Tuomas Sandholm, and Subhash Suri. Solving combinatorial exchanges: Optimality via a few partial bids. In 3rd International Joint Conference on Autonomous Agents and Multiagent Systems (AAMAS 2004), 19-23 August 2004, New York, NY, USA, pages 1418-1419. IEEE Computer Society, 2004.

[24] Peep Küngas and Mihhail Matskin. Symbolic negotiation with linear logic. In Jürgen Dix and João Leite, editors, Computational Logic in Multi-Agent Systems, pages 71-88, Berlin, Heidelberg, 2005. Springer Berlin Heidelberg.

[25] Vadim Levit, Tal Grinshpoun, Amnon Meisels, and Ana L.C. Bazzan. Taxation Search in Boolean Games. In 12th International Conference on Autonomous Agents and Multi-agent Systems, AAMAS'13, pages 183-190. International Foundation for Autonomous Agents and Multiagent Systems, 2013.

[26] Patrick Lincoln, John Mitchell, Andre Scedrov, and Natarajan Shankar. Decision problems for propositional linear logic. Annals of Pure and Applied Logic, 56(1-3):239-311, 1992.

[27] Dov Monderer and Lloyd S. Shapley. Potential games. Games and Economic Behavior, 14(1):124 $-143,1996$.

[28] Pavel G. Naumov and Jia Tao. A modal logic for reasoning about economic policies. Journal of Logic and Computation, 27(1):395-412, 2017.

[29] Peter W. O'Hearn and David J. Pym. The logic of Bunched Implications. Bulletin of Symbolic Logic, 5(2):215-244, 1999.

[30] Martin J. Osborne and Ariel Rubinstein. A course in game theory. The MIT Press, Cambridge, USA, 1994.

[31] Christos Papadimitriou. Computational Complexity. Addison Wesley, 1994. 
[32] Christos Papadimitriou and Mihalis Yannakakis. The complexity of facets (and some facets of complexity). Journal of Computer and System Sciences, 28(2):244-259, 1984.

[33] Daniele Porello and Ulle Endriss. Modelling Combinatorial Auctions in Linear Logic. In Fangzhen Lin, Ulrike Sattler, and Miroslaw Truszczynski, editors, Principles of Knowledge Representation and Reasoning: Proceedings of the Twelfth International Conference, KR 2010, pages 71-78. AAAI Press, 2010.

[34] Daniele Porello and Ulle Endriss. Modelling Multilateral Negotiation in Linear Logic. In ECAI 2010 - 19th European Conference on Artificial Intelligence, Proceedings, volume 215 of Frontiers in Artificial Intelligence and Applications, pages 381-386. IOS Press, 2010.

[35] Daniele Porello and Nicolas Troquard. Non-normal modalities in variants of linear logic. Journal of Applied Non-Classical Logics, 25(3):229-255, 2015.

[36] Torsten Reiners and Lincoln C. Wood, editors. Gamification in Education and Business. Springer, Cham, 2015.

[37] Greg Restall. On Logics Without Contraction. PhD thesis, The University of Queensland, 1994.

[38] John C. Reynolds. Separation Logic: A Logic for Shared Mutable Data Structures. In Proceedings of the 17th Annual IEEE Symposium on Logic in Computer Science, LICS'02, pages 55-74, Washington, DC, USA, 2002. IEEE Computer Society.

[39] Robert W. Rosenthal. A class of games possessing pure-strategy Nash equilibria. International Journal of Game Theory, 2(1):65-67, Dec 1973.

[40] Jörg Rothe. Complexity Theory and Cryptology. Springer-Verlag Berlin Heidelberg, 2005.

[41] Anne S. Troelstra. Lectures on Linear Logic. CSLI Publications, 1992.

[42] Nicolas Troquard. Nash equilibria and their elimination in resource games. In Proceedings of the Twenty-Fifth International Joint Conference on Artificial Intelligence, IJCAI 2016, pages 503-509. AAAI Press, 2016.

[43] Nicolas Troquard. Rich coalitional resource games. In Proceedings of the Thirty-Second AAAI Conference on Artificial Intelligence, AAAI 2018, pages 1242-1249. AAAI Press, 2018.

[44] Yaron Velner, Krishnendu Chatterjee, Laurent Doyen, Thomas A. Henzinger, Alexander Moshe Rabinovich, and Jean-François Raskin. The complexity of multi-mean-payoff and multi-energy games. Inf. Comput., 241:177-196, 2015.

[45] Klaus W. Wagner. More Complicated Questions About Maxima and Minima, and Some Closures of NP. Theor. Comput. Sci., 51(1-2):53-80, March 1987.

[46] Gerd Wechsung. On the boolean closure of NP. In Lothar Budach, editor, Fundamentals of Computation Theory, volume 199 of Lecture Notes in Computer Science, pages 485-493. Springer Berlin Heidelberg, 1985.

[47] Michael Wooldridge and Paul E. Dunne. On the computational complexity of coalitional resource games. Artificial Intelligence, 170(10):835-871, 2006. 